\title{
Decimetric Emission 500" Away from a Flaring Site: Possible Scenarios from GMRT Solar Radio Observations
}

\author{
Susanta Kumar Bisoi ${ }^{1}$ (D) H. S. Sawant ${ }^{2}$, P. Janardhan ${ }^{3}$ (i), Y. Yan ${ }^{1}$, L. Chen ${ }^{1}$, Arun Kumar Awasthi ${ }^{4}$ (D), Shweta Srivastava ${ }^{3}$, and \\ G. $\mathrm{GaO}^{5}$ \\ ${ }^{1}$ Key Laboratory of Solar Activity, National Astronomical Observatories, Chinese Academy of Sciences, Beijing 100012, People's Republic of China \\ susanta@nao.cas.cn \\ ${ }^{2}$ INPE-National Institute for Space Research, Sao Jose dos Campos, SP, Brazil \\ ${ }^{3}$ Astronomy \& Astrophysics Division, Physical Research Laboratory, Ahmedabad 380 009, India \\ ${ }^{4}$ Key Laboratory of Geospace Environment, Department of Geophysical and Planetary Sciences, University of Science and Technology of China, Hefei 230026 , \\ People's Republic of China \\ ${ }^{5}$ Yunnan Observatories, Chinese Academy of Sciences, Kunming 650011, People's Republic of China \\ Received 2016 November 26; revised 2018 June 14; accepted 2018 June 14; published 2018 July 24
}

\begin{abstract}
We present a study of decimetric radio activity using the first high time cadence $(0.5 \mathrm{~s})$ images from the Giant Meterwave Radio Telescope (GMRT) at $610 \mathrm{MHz}$ associated with GOES C1.4- and M1.0-class solar flares and a coronal mass ejection (CME) onset that occurred on 2015 June 20. The high spatial resolution images from GMRT show a strong radio source during the $\mathrm{C} 1.4$ flare located $\sim 500^{\prime \prime}$ away from the flaring site with no corresponding bright footpoints or coronal features nearby. In contrast, however, strong radio sources are found near the flaring site during the M1.0 flare and around the CME onset time. Weak radio sources located near the flaring site are also found during the maximum of the C1.4 flare activity that show a temporal association with metric Type III bursts identified by the Solar Broadband Radio Spectrometer at Yunnan Astronomical Observatory. Based on a multiwavelength analysis and magnetic potential field source surface extrapolations, we suggest that the source electrons of GMRT radio sources and metric Type III bursts originated from a common electron acceleration site. We also show that the strong GMRT radio source is generated by a coherent emission process, and its apparent location far from the flaring site is possibly due to the wave-ducting effect.
\end{abstract}

Key words: Sun: flares - Sun: particle emission - Sun: radio radiation

Supporting material: animations

\section{Introduction}

Decades of observations have shown that solar flares release energy as large as $10^{23}-10^{33}$ erg via the process of magnetic reconnection, thereby heating the coronal plasma and accelerating particles (e.g., Priest \& Forbes 2000; Aschwanden 2002; Benz 2008; Fletcher et al. 2011). The accelerated electrons, while propagating in the corona, emit in hard X-ray (HXR) and radio wavelengths at different heights, depending on the electron energy and coronal electron density. However, an intriguing question remains regarding the location of the electron acceleration. Several earlier studies arrived at a consensus that the primary electron acceleration sites are located in the lower corona (Bastian et al. 1998; White et al. 2011), where the electron density $n_{e}$ is $10^{9}-10^{10} \mathrm{~cm}^{-3}$. The radio emission detected from this region, if due to plasma radiation, usually occurs in the decimeter wavelength range, i.e., $f \approx 500-2000 \mathrm{MHz}$ (Aschwanden 2002, 2005). It is, therefore, of particular interest in utilizing the decimetric flare emission to locate and diagnose the electron acceleration sites and, in turn, the flare energy release region.

Solar radio emission at decimetric and longer wavelengths is broadly categorized into five types-Types I, II, III, IV, and V (Melrose 1980; Dulk 1985) —and it usually shows a very high brightness temperature $\left(>10^{9} \mathrm{~K}\right)$ due to its generation by a coherent emission mechanism (Dulk 1985). In a plasma radiation process, plasma Langmuir waves are excited by accelerated nonthermal electrons. They are converted to radio emission at the fundamental $\left(f_{p}=8980 \sqrt{n_{e}}\right.$, where $f_{p}$ is in $\mathrm{Hz}$ and $n_{e}$ is in $\mathrm{cm}^{-3}$ ) and/or harmonic $\left(2 f_{p}\right)$ frequencies (Wild et al. 1953; Wild \& Smerd 1972; Melrose 1980; Dulk 1985; Bastian et al. 1998). Another possible coherent emission mechanism is the electron cyclotron maser (ECM) radiation process (Melrose \& Dulk 1982). It has been proposed in order to explain narrowband $(10-150 \mathrm{MHz})$, small source size $\left(3 \times 10^{9} \mathrm{~cm}\right)$, strong circular polarization $(100 \%)$, and high brightness temperature $\left(>10^{12} \mathrm{~K}\right)$ of spike bursts usually accompanying Type IV bursts (Droege 1977; Slottje 1980). Spike bursts that accompany Type III bursts (Benz et al. 1982; Benz 1985), however, show low to intermediate circular polarization. Generally, for ECM emission to be possible, it requires that $f_{p}$ must be less than $f_{b}\left(=\frac{e B}{2 \pi m_{e}}\right.$, where $f_{b}$ and $B$ are, respectively, electron cyclotron frequency and magnetic field, and $e$ and $m_{e}$ are, respectively, charge and mass of electron) at the radio emission site (Melrose \& Dulk 1982; Melrose 1991).

Using high time sampling spectrograph and/or imaging observations, decimetric flare emissions including fast-drift Type III bursts (Aschwanden \& Benz 1995; Benz 2004, 2008 and references therein; Chen et al. 2013), drifting pulsating structures (DPSs; Kliem et al. 2000; Karlický et al. 2002; Karlický 2004; Karlický \& Bárta 2007), and short-duration narrowband spikes (Droege 1977; Benz et al. 1982; Benz 1985, 2004; Tan 2013; Chen et al. 2015) have been recognized and studied. The escape of radio waves at decimetric wavelengths from the flare site is, however, difficult due to the usually strong free-free absorption by the relatively dense plasma in the low corona (Aschwanden \& Benz 1995; Aschwanden 2002). A more favorable condition for detecting radio sources at this wavelength range requires relatively weak 
free-free absorption along its way (Aschwanden 2002), which can be achieved when the energetic source electrons are injected into overdense structures filled with hot coronal plasma or the radio waves are ducted along a density-depleted magnetic flux tube outward. In the low-beta corona, both open magnetic flux tubes and high-arching closed loops can act as density-depleted ducts (Duncan 1979). The ducting of streaming electrons generally causes a shift in the position of the true radio source, which was first commonly seen in the singlefrequency records of Culgoora heliograph radio images (Sheridan et al. 1972; Stewart 1972; Duncan 1979). The true source is located at a lower coronal height, where the radio wave is actually generated, than that of the apparent source in the radio image. The trapped radio waves only leave the flux tube at the apparent source position when the wave frequency inside the tube is greater than the plasma frequency outside. Such displacements of the apparent radio source have also been seen in radio images from the LOw Frequency ARray (LOFAR; Kontar et al. 2017; Mann et al. 2018) at metric wavelengths. Based on a study of fine structures of Type III radio bursts using LOFAR observations, Kontar et al. (2017) suggested that the apparent source locations of fundamental and harmonic emission result from the scattering of radio waves by coronal density inhomogeneities. Recently, using LOFAR observations, Mann et al. (2018) reported Type III radio bursts located higher up in the corona and showing nonuniform movements in their source positions. The authors suggested that the Type III radio bursts were due to plasma radiation and the apparent shifts in their locations were caused by coronal propagation effects. In another study using Nancay Radio Heliograph (NRH) images at $450 \mathrm{MHz}$, Benz et al. (2002) reported low-frequency decimetric spikes that were located about 20"-200" away from the HXR and soft X-ray (SXR) sources.

In an earlier study of radio emission at a decimetric wavelength range using the GMRT at $610 \mathrm{MHz}$, Kundu et al. (2006) also reported radio sources located about 200" from the flaring active region. In the present paper, using the first high time cadence images at $610 \mathrm{MHz}$ from the GMRT, we have studied the decimetric radio activity associated with $\mathrm{C}$ - and M-class flares and a coronal mass ejection (CME) onset that occurred on 2015 June 20 and reported a decimetric radio source during the C-class flare to be located about 500" away from the flaring site. The time cadence of the present GMRT observations is $0.5 \mathrm{~s}$, while the earlier reported GMRT observations had a maximum time cadence of $16.9 \mathrm{~s}$ (Subramanian et al. 2003), 2.11 s (Kundu et al. 2006), or $1 \mathrm{~s}$ (Mészárosová et al. 2013). The high time cadence images of GMRT $610 \mathrm{MHz}$ during the C-class flare have also been used, in combination with the coronal images of extreme ultraviolet (EUV) and X-ray wavelengths, to investigate the genesis of radio-emitting electrons of decimetric sources and their temporal association with the Type III burst activity at metric wavelengths. The rest of the paper is organized as follows. Section 2 briefly discusses solar observations at the GMRT. In Section 3, observations and analyses of GMRT $610 \mathrm{MHz}$ data on 2015 June 20 and the associated data at EUV, X-ray, and metric wavelengths are discussed. Subsequently, in Section 4, we report the spatiotemporal distribution, polarization, and magnetic configuration of GMRT $610 \mathrm{MHz}$ sources and the association with metric Type III bursts. Finally, Sections 5 and 6 interpret and conclude our results.

\section{Instrument}

\subsection{The GMRT}

The GMRT (Swarup et al. 1991) is a radio interferometer and consists of $3045 \mathrm{~m}$ diameter antennas spread over a radius of $25 \mathrm{~km}$. It is located in India, about $80 \mathrm{~km}$ from Pune. Fourteen antennas are in a compact configuration, randomly distributed in a central square area of $1 \mathrm{~km}$, while the remaining antennas are distributed along three arms in a $\mathrm{Y}$ shape. The shortest baseline at GMRT is $100 \mathrm{~m}$, while the longest baseline is $25 \mathrm{~km}$. The long baselines of GMRT offer a high spatial resolution (up to $\sim 5^{\prime \prime}$ at $610 \mathrm{MHz}$ ), making it one of the most important interferometers operating in the low-frequency range $(150-1400 \mathrm{MHz})$. The GMRT actually observes at six distinct frequencies: 150, 236, 327, 610, 1280, and $1400 \mathrm{MHz}$. Though the GMRT is not a dedicated solar telescope, it has been used successfully for observing and studying the Sun (Subramanian et al. 2003; Kundu et al. 2006; Mercier et al. 2006; Mészárosová et al. 2013; Mercier et al. 2015). Snapshot solar images with unprecedented resolution and high dynamic range, far better than any images previously obtained, have been produced by combining visibilities from the GMRT and NRH (Mercier et al. 2006). This technique has been used by Mercier et al. (2015) to study the fine structures of solar noise storms using joint observations of the GMRT and NRH and has achieved resolutions as small as $30^{\prime \prime}$ at $327 \mathrm{MHz}$ and $35^{\prime \prime}$ at $236 \mathrm{MHz}$. Only a few studies have been reported at GMRT $610 \mathrm{MHz}$. The first study (Kundu et al. 2006) of GMRT $610 \mathrm{MHz}$ radio emission reported an arc-shaped radio source morphology of decimetric flare emission, describing its origin as due to trapped radiating electrons at the magnetic loop tops. In another study of GMRT $610 \mathrm{MHz}$ (Mészárosová et al. 2013), during a weak B8.9 flare, the authors reported very weak radio sources located in the fan structure of coronal magnetic field lines originating from a coronal magnetic null point.

The observational procedure during solar observations at GMRT has been described in Subramanian et al. (2003) and Mercier et al. (2006). For the present GMRT solar observation of 2015 June 20, the Sun was continuously observed first for $1 \mathrm{hr}$, after which a nearby phase calibrator was observed for around 7 minutes. This cycle of Sun and phase calibrator observations was repeated during the entire allotted observation period. During each Sun scan, the antennas were positioned at the Sun center at the start of the scan, and tracking was carried out at a linear rate in both right ascension and declination. In subsequent scans, the antennas were again repositioned to the Sun center at the start of the scan and tracked as before. In addition to the phase calibrator observations, a strong flux calibrator (such as 3C147) was also observed at both the start and the end of the observation.

\section{Observation and Data Analysis}

A GOES M-class (M1.0) and two GOES C-class (C2.3 and C1.4) solar flares were recorded on 2015 June 20 as seen from GOES15 SXR observations (Figure 1, top). The C2.3 flare was a short-duration flare that started at 02:34:41 UT and ended at 02:44:00 UT, while the C1.4 flare was a rather long-duration flare that lasted for over $2 \mathrm{hr}$. The $\mathrm{C} 1.4$ flare started at 03:14:41 UT and ended at 04:50:00 UT and was followed by an M1.0 flare that started at 05:57:21 UT and lasted for only 20 minutes. The onset time of the flares is determined by the time when the 

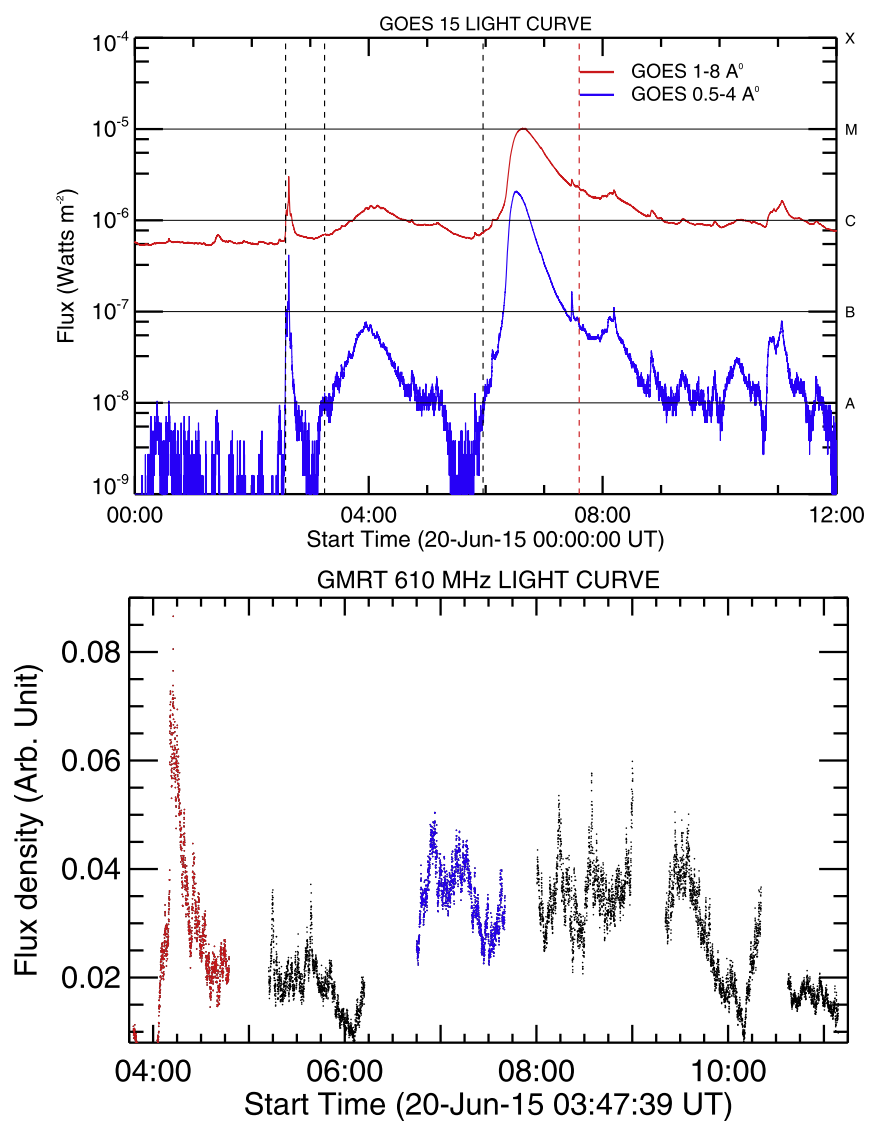

Figure 1. (Top) Temporal profile of GOES15 X-ray observations on 2015 June 20 . The vertical black dotted lines indicate the onset of GOES C2.3-, C1.4- and M1.0-class solar flares, respectively, at 02:34:41, 03:14:41, and 05:57:21 UT. The vertical red dotted line indicate the onset of the CME in the LASCO/C2 field of view at 07:36 UT. (Bottom) The GMRT $610 \mathrm{MHz}$ light curve for the period 03:47:39-11:09:16 UT, covering the period of a C1.4 flare duration (indicated by a dotted red curve) and an M1.0 flare and a CME (indicated by a dotted blue curve).

SXR flux is above the $3 \sigma$ level of the pre-flare level. The vertical black dotted lines in the top panel of Figure 1 demarcate the onset of the respective flares. Just after the M1.0class flare, a CME erupted at 07:36:00 UT, as recorded in the C2 field of view of the Large Angle and Spectrometric Coronagraph (LASCO), and is indicated by a vertical red dotted line in the top panel of Figure 1.

The GMRT radio observations of 2015 June 20 were recorded from 03:47:39 to 11:09:16 $\mathrm{UT}$ at $610 \mathrm{MHz}$ with a time cadence of $0.5 \mathrm{~s}$, covering the C1.4 and M1.0 flares and the CME. The observed GMRT data were first properly edited and calibrated using both flux and phase calibrators. The calibrated data during the flare intervals were then processed using a standard clean procedure in the Astronomical Image Processing System, a standard radio astronomy software package used primarily for data reduction of astronomical radio data sets. Generally, images so produced are registered properly; however, in order to make the image registration proper, we applied an additional calibration. The calibration was carried out based on self-calibration solutions obtained at the peak of the burst during the M1.0 flare. The solutions were then applied to the radio data of both the C1.4 and the M1.0 flares, and cleaned images were finally produced. Figure 1 (bottom) shows the GMRT $610 \mathrm{MHz}$ light curve during the period 03:47:39-11:09:16 UT. The light curve was obtained from GMRT snapshot images made at $2 \mathrm{~s}$ intervals. From Figure 1 (bottom), a strong flux enhancement during GMRT observations of the $\mathrm{C} 1.4$ flare period (indicated by a dotted red curve) is clearly evident in comparison to the flux enhancements during GMRT observations of the M1.0 flare and the CME (indicated by a dotted blue curve).

For GMRT solar observations, an attenuation of $30 \mathrm{~dB}$ is usually used, so the absolute amplitudes of solar observations at GMRT are not properly obtained. Hence, in order to estimate the absolute flux of the present GMRT $610 \mathrm{MHz}$ observations, we compared the $610 \mathrm{MHz}$ flux at GMRT to the solar flux level at $610 \mathrm{MHz}$ obtained by the patrol telescopes of the Radio Solar Telescope Network (RSTN) at the Learmonth station. The flux levels at both GMRT and RSTN for the C1.4 flare show a similar time structure, from 04:07:12 to 04:16:48 UT, around the radio flare maximum (figure not shown here). So, we computed a scaling factor of 4000 to normalize the GMRT $610 \mathrm{MHz}$ flux with respect to the RSTN $610 \mathrm{MHz}$ flux, covering the $\mathrm{C} 1.4$ flare observation period recorded at GMRT.

We used EUV observations from the AIA instrument (Lemen et al. 2012) on board the SDO (Pesnell et al. 2012) and X-ray observations from the Reuven Ramaty High Energy Solar Spectroscopic Imager (RHESSI; Lin et al. 2002). Full disk images from the selected AIA passband with high temporal (12 s) and spatial ( 0 ". 6$)$ resolution centered on six EUV wavelengths $(94,131,171,193,211$, and $335 \AA$ ) were used, along with the line-of-sight (LOS) magnetograms from the Helioseismic Magnetic Imager (HMI; Schou et al. 2012) having a time cadence of $45 \mathrm{~s}$ and a spatial resolution of 0 ". 6 . RHESSI images for different energy bands, spanning the range 6-50 keV, were reconstructed using the CLEAN method (Hurford et al. 2002) with a spatial resolution of 2!.3. The integration time for the RHESSI images is 1 minute when the RHESSI count rate is low and $20 \mathrm{~s}$ when the count rate is high. All EUV, X-ray, and radio images during the C1.4 and M1.0 flare observation periods were derotated to their respective radio flare peak at GMRT $610 \mathrm{MHz}$ to account for the effects of the solar differential rotation.

In addition, the radio dynamic spectra (the solar flux distributions in the frequency-time plane) at low frequencies were obtained from the Solar Broadband Radio Spectrometer at Yunnan Astronomical Observatory (SBRS/YNAO; Gao et al. 2014). The YNAO dynamic spectra used were comprised of metric spectrograph observations in the frequency range $70-700 \mathrm{MHz}$ in both left and right circular polarizations (LCPs and RCPs). The YNAO data have a spectral resolution of $0.2 \mathrm{MHz}$ and a temporal resolution of $80 \mathrm{~ms}$. However, due to severe interference in channels below $130 \mathrm{MHz}$ and above $300 \mathrm{MHz}$, we presented only the interference-free metric spectra in the frequency range $130-300 \mathrm{MHz}$.

\section{Results}

\subsection{Spatial Distribution of GMRT $610 \mathrm{MHz}$ Radio Sources}

Snapshot images at GMRT $610 \mathrm{MHz}$ at the flare maximum of the C1.4 and M1.0 flares and the onset of the CME are shown in Figure 2 (top). The images have a clean beam of the order of $12^{\prime \prime}$, indicated in the bottom left corner in the top row of panels of Figure 2. The signal-to-noise ratio at the peak of the burst during the C1.4 and M1.0 flares was 306 and 377, respectively, and the background noise level during the low flux periods was $3.5 \times 10^{5} \mathrm{~K}$. The middle row of Figure 2 

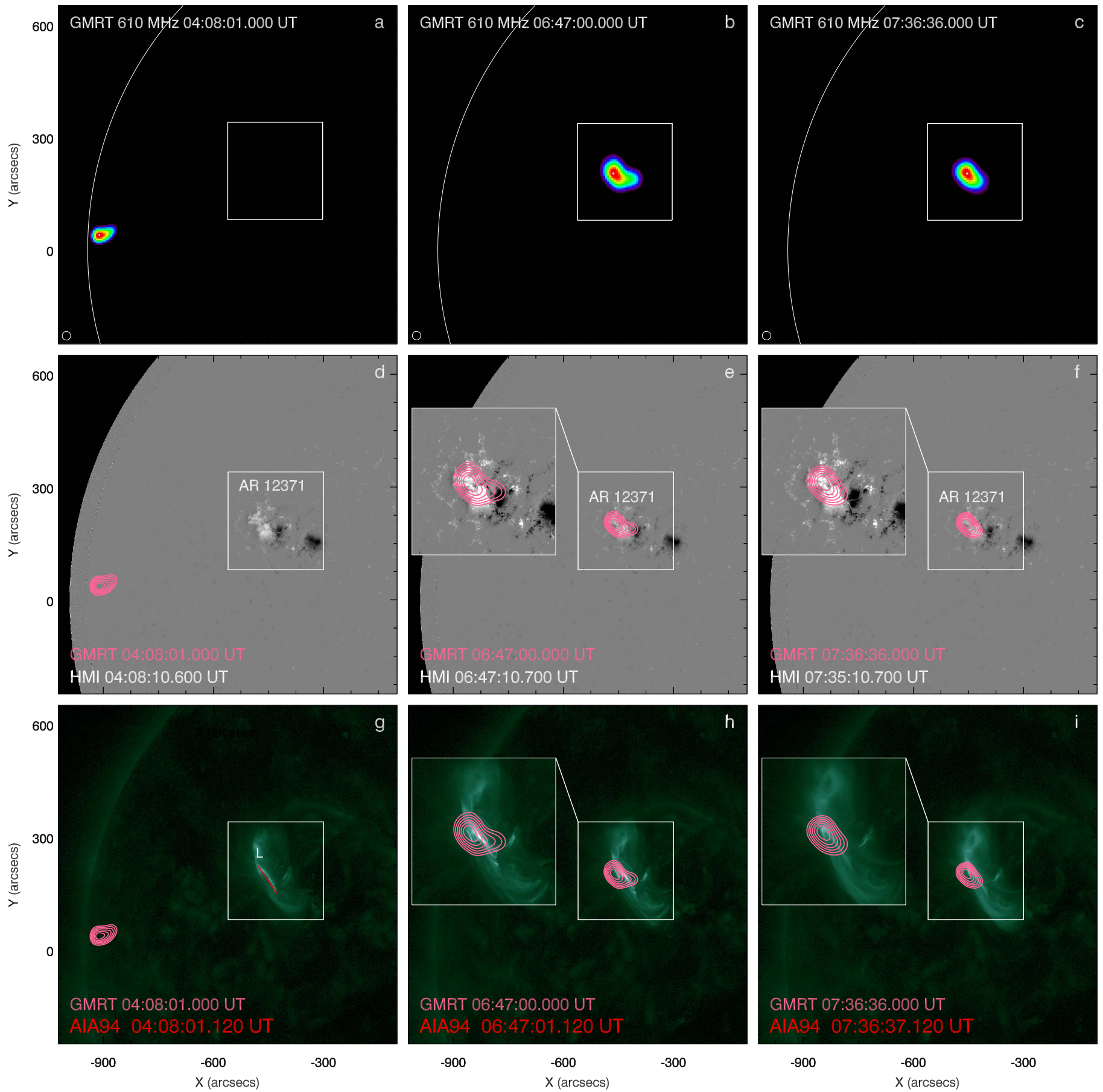

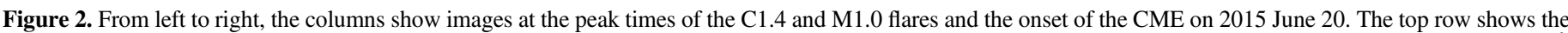

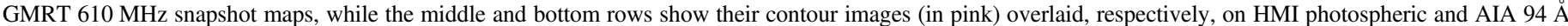
EUV images.

shows GMRT $610 \mathrm{MHz}$ contours in pink overplotted on background SDO/HMI images. The contour levels are drawn at $40 \%, 50 \%, 60 \%, 70 \%, 80 \%$, and $90 \%$ of the maximum brightness temperature in each image. The SDO/AIA $94 \AA$ images overplotted by GMRT $610 \mathrm{MHz}$ contours, in pink, are shown in the bottom row of Figure 2.

A radio source located above the flaring active region, AR12371, at the time of the M1.0 flare maximum and CME onset is seen in Figures 2(b) and (c). In contrast, a radio source at the $\mathrm{C} 1.4$ flare maximum is seen located near the southeast limb, about $500^{\prime \prime}$ or $365,000 \mathrm{~km}$ away from the flaring active region. No other active regions are seen near the location of this radio source from $S D O / \mathrm{HMI}$ images (middle row) or any corresponding coronal features such as coronal loops from SDO/AIA $94 \mathrm{~A}$ images (bottom row). This raises the question as to whether the observed limb radio source during the $\mathrm{C} 1.4$ flare is real. Our analysis, which shows radio sources located near the flaring active region and cospatial with the hot coronal loops (middle and bottom rows) during the M1.0 flare and CME, suggests that the image registration is proper. Thus, we conclude that the radio source observed during the $\mathrm{C} 1.4$ flare is real. 
It is found that the electron plasma density in the corona usually varies in the range $1.2-4.6 \times 10^{9} \mathrm{~cm}^{-3}$ if the radiation is due to fundamental and harmonic plasma radiation at a frequency of $610 \mathrm{MHz}$. The electron plasma density above active regions generally falls in this range, and so one expects that the GMRT $610 \mathrm{MHz}$ radio source would be located near the active region, which is actually not the case for the GMRT $610 \mathrm{MHz}$ source observed during the period of the C1.4 flare. Hence, it is interesting and unique, and henceforth, we mainly focus on the nature and origin of this GMRT radio source and the study of GMRT radio observations associated with only the C1.4 flare period. A detailed analysis of GMRT $610 \mathrm{MHz}$ observations of the M1.0 flare and CME evolution will be described in a subsequent paper.

\subsection{Spatial Distributions of GMRT $610 \mathrm{MHz}$ Sources during the C1.4 Flare}

Figure 3(a) shows a GMRT $610 \mathrm{MHz}$ map during the radio flare maximum at 04:11:05 UT (an animation of Figure 3(a) that shows GMRT $610 \mathrm{MHz}$ snapshots at $2 \mathrm{~s}$ interval from 04:02:45 to 04:11:59 UT, covering the rise to peak phase of the $610 \mathrm{MHz}$ radio flare, is available in the online version of the journal). As discussed earlier, a radio source, labeled as LS, is located to the southeast limb. The source first appeared at 04:02:45 UT (see the online version of Figure 3(a)) having a low source flux density. With time, the source flux density increases, and a strong flux density is observed from 04:10:23 UT. At the same time, weak sources located near the flaring active region AR12371 are also observed (see the online version of Figure 3(a)). The weak sources are well resolved by the GMRT clean beam and shown in the inset of Figure 3(a) labeled as S1, S2, and S3. The flux densities of the weak sources are above the background noise level, and hence we consider them as real.

We estimated the sizes of GMRT sources by using a twodimensional Gaussian fit to the full width at half maximum of source emission regions and found that sources S1 and S2 have apparent angular sizes of $\sim 20^{\prime \prime}$, or source dimensions of $1.4 \times 10^{9} \mathrm{~cm}$. Similarly, source S3 has an apparent angular size of $\sim 10^{\prime \prime}$, or $7.2 \times 10^{8} \mathrm{~cm}$. Source LS is found to have an apparent angular size of $33^{\prime \prime} \times 20^{\prime \prime}$, or source dimensions of $2.3 \times 10^{9} \times 1.4 \times 10^{9} \mathrm{~cm}$. We computed the brightness temperature of all GMRT radio sources following the method described in Subramanian et al. (2007), which is indicated by color bars shown in Figure 3(a). All of the GMRT sources have a high brightness temperature $>10^{11} \mathrm{~K}$, with source LS having a brightness temperature of $\sim 1.4 \times 10^{12} \mathrm{~K}$, about an order of magnitude higher than that of sources S1, S2, and S3.

The overplotted GMRT source contours on the LOS photospheric HMI magnetogram are shown in Figure 3(b). (An animation of Figure 3(b) showing the overlaid GMRT source contours on HMI images from 04:02:45 to 04:11:59 UT is available in the online version of the journal.) The $\mathrm{C} 1.4$ solar flare occurred in active region AR12371, the location of which is indicated by a white cross in Figures 3(b)-(d). The active region AR12371 is shown in the inset of Figure 3(b). It is a complex active region having pairs of sunspots of positive polarity, indicated by $\mathrm{P} 1$ and $\mathrm{P} 2$, and negative polarity, indicated by $\mathrm{N} 1$ and N2. From the overlaid GMRT source contours on the HMI images, it can be seen that the source S1 is located above N1, while the source S3 is located above P1. The source S2 is displaced southward, about $40^{\prime \prime}$ to the east of the flare location, with no corresponding magnetic features in the magnetogram. The source LS, however, has shown even further displacement of about 500".

The GMRT source contours overplotted on coronal images from SDO/AIA are shown in Figures 3(c) and (d). Figure 3(c) shows a composite image constructed from AIA hot passbands of 94, 131, and $335 \AA$, while Figure 3(d) shows a composite image constructed from AIA cold passbands of 211, 193, and $171 \AA$. A partially formed sigmoid is visible from the AIA hot channel image. The sigmoid is visible in the AIA cold channel image after the $\mathrm{C} 1.4$ flare maximum. At the northern end of the sigmoid, we can see hot coronal loops present near the flare location.

We prepared RHESSI clean images in 6-12 and $12-25 \mathrm{keV}$ energy bands corresponding to the period 04:10-04:12 UT, when the weak GMRT sources have been identified near the flaring active region. An animation of the RHESSI clean images in the 6-12 and $12-25 \mathrm{keV}$ energy bands is available in the online version of Figure 4. From RHESSI 6-12 keV animation, an SXR source is clearly visible during the entire period. However, no consistent source structure is seen in the RHESSI $12-25 \mathrm{keV}$ images during the same period. A contour of the high-resolution ( $\sim \mathrm{s})$ RHESSI $6-12 \mathrm{keV}$ image (in violet) made at 04:11:05 UT is also overlaid in the inset maps of AIA hot and cold channel images of Figures 3(c) and (d) and shows a clear SXR source. But no HXR sources are seen in the highresolution RHESSI 12-25 keV image made at 04:11:05 UT, and hence no HXR footpoints are observed (within the RHESSI sensitivity). It is seen from Figures 3(c) and (d) that the radio sources S1 and S3 are nearly cospatial with the SXR source. Also, as seen from Figure 3(b), the sources S1 and S3 lie on enhanced magnetic field regions. This suggests that the sources $\mathrm{S} 1$ and $\mathrm{S} 3$ could be radio sources located near the flaring active region. The sources $\mathrm{S} 2$ and $\mathrm{LS}$, on the other hand, are located far from the SXR source. Also, when the sources S2 and LS are projected on the HMI magnetogram (Figure 3(b)), we find no magnetic field enhanced regions, indicating they could not be located near the flaring active region. They presumably could be located on tall long loops (Kundu et al. 2006) and at relatively higher heights in comparison to the sources S1 and S3.

\subsection{Temporal Evolution of GMRT $610 \mathrm{MHz}$. Sources during the C1.4 Flare}

The temporal evolution of the radio fluxes of all of the GMRT $610 \mathrm{MHz}$ sources during the GOES C1.4 flare are shown in panel (a) of Figure 5. The radio light curves of all of the sources were obtained from GMRT solar maps by averaging solar fluxes from the surrounding region of the respective sources. The radio light curve of source LS (black curve in panel (a) of Figure 5) shows a flux level enhancement starting from 04:02:45 UT that continues to increase until around 04:10:23 UT, just when an abrupt enhancement in its radio flux level is noticed. Subsequently, its flux level shows a first peak during the radio flare maximum, indicated by a vertical gray bar in the left panels of Figure 5. A second peak (maximum) is seen at 04:12:37 UT. Source S1 (red curve in panel (a) of Figure 5) shows a similar variation in its flux level, like source LS, with an abrupt enhancement in its flux shortly after 04:10:25 UT and afterward peaking at 04:10:47 UT. The fluxes for sources S2 (blue curve in panel (a) of Figure 5) and S3 (light red curve) are also enhanced during this period, peaking at 04:11:08 and 04:10:33 UT, respectively. The fluxes 

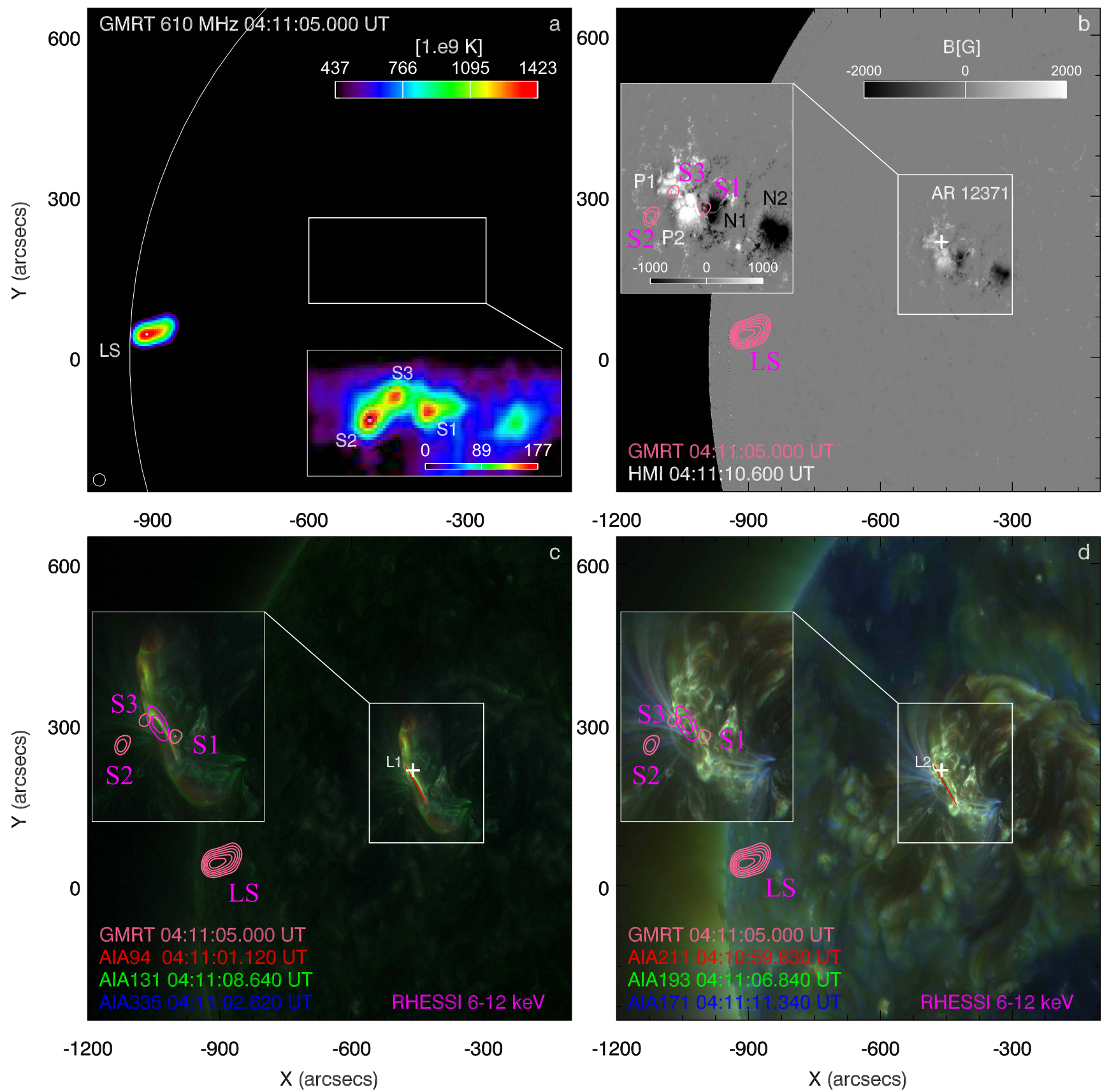

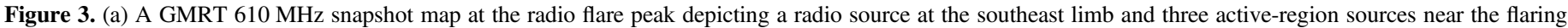

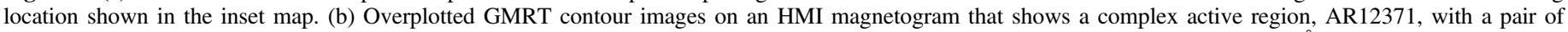

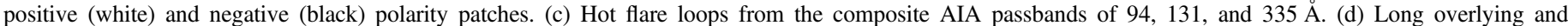

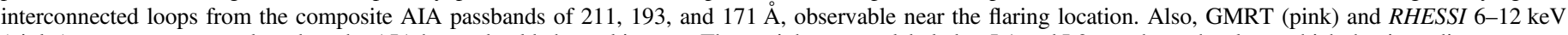

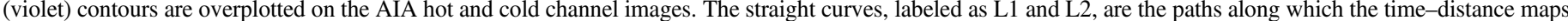

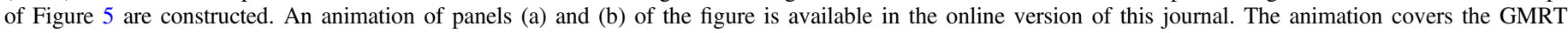
$610 \mathrm{MHz}$ images from 04:02:45 to 04:11:59 UT, while the static figure displays this data at 04:11:05 UT.

(An animation of this figure is available.)

of sources S2 and S3, after the radio flare maximum, decline within a few minutes, while the fluxes of sources S1 and LS gradually decline, showing a steady emission in the flare decline phase. It is noted that a burst peak is present in all of the GMRT sources during the $610 \mathrm{MHz}$ flare maximum, although they show different peak times. This suggests that the source electrons of all of the GMRT sources could have access to a common electron acceleration site. The different peak flux times of all of the sources, however, indicate that they could be located in different loop systems, which is apparent from their different spatial locations, as seen in Figure 3.

Enhanced X-ray and EUV emission is evident during the $610 \mathrm{MHz}$ flare, as noted from GOES15, RHESSI, and SDO/AIA (94 and $131 \AA$ A passbands) light curves (Figures 5(b) and (c)). There is, however, no one-to-one temporal correspondence between $610 \mathrm{MHz}$ and X-ray fluxes (GOES and RHESSI $6-12 \mathrm{keV}$ ) during the radio flare maximum. This is commonly noticed in the case of decimetric frequency bursts 


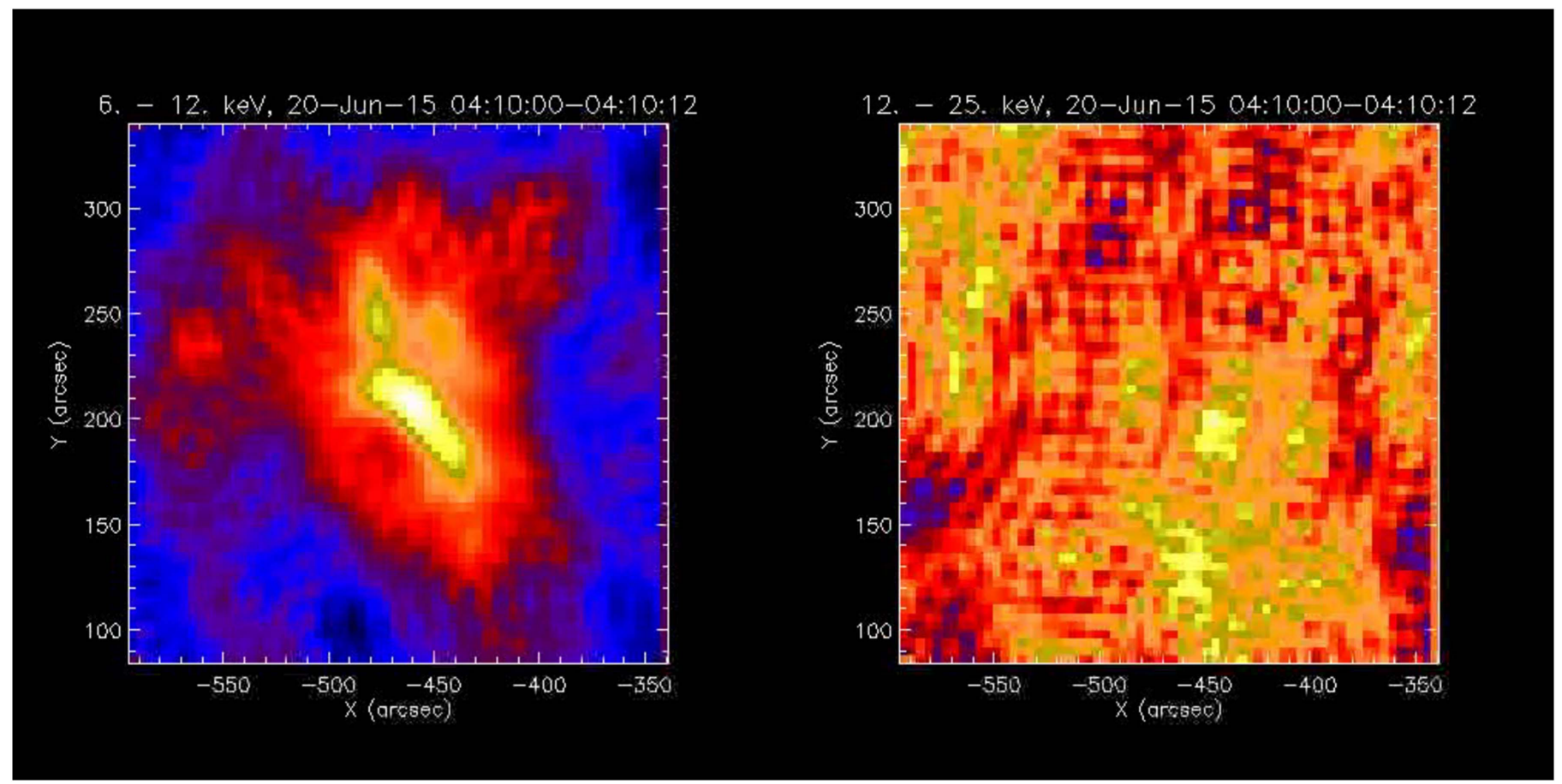

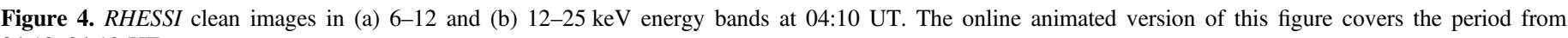
04:10-04:12 UT.

(An animation of this figure is available.)

(Benz et al. 1983). It is observed that a RHESSI 6-12 keV $\mathrm{X}$-ray burst occurred about 2 minutes prior to the radio flare peak. Such a delay in the radio to SXR emission has been noted (Aschwanden \& Benz 1995) for decimetric Type III bursts that peaked few minutes after the SXR burst. Very low counts in the RHESSI 12-25 keV energy band and hardly any counts in the RHESSI 25-50 keV (not shown here) energy band indicate that there is very weak/no nonthermal X-ray emission (within the sensitivity of the RHESSI instrument) around the radio flare maximum.

The distance-time plots were constructed by extracting the EUV intensity from AIA hot and cold channel images along the straight curve paths labeled L1 and L2 in Figures 3(c) and (d), respectively. A first flare loop brightening is observable at 03:50:20 UT from the distance-time plots of AIA hot and cold channel images (Figures 5(d) and (e)), while the flare loops brightened later between 04:07:25 and 04:10:25 UT, as seen only in hot channel images (Figure 5(d)), corresponding to the $610 \mathrm{MHz}$ radio flare maximum period when the burst peak in all of the GMRT sources has been observed.

In order to determine the thermal and nonthermal characteristics of the flare plasma during the radio flare maximum, we analyzed the RHESSI X-ray spectra. We prepared a time series of background-subtracted X-ray spectra during 04:10-04:12 UT at a time cadence of $12 \mathrm{~s}$. The background was determined by averaging the observations during 01:18:45-01:20:25 UT, corresponding to the closest-in-time quiet period. From the RHESSI X-ray count rates (figure not shown here), we find that the RHESSI count rates have been strongly affected by the particle emission, particularly in the energy bands $>50 \mathrm{keV}$. Thus, in order to indemnify the effect of high-energy particles, the time profile of the background was set to the "high-E profile" option provided in the Object Spectral Energy Executive (OSPEX) package within the SolarSoft distribution.
This option makes use of the intensity profile recorded in the highest energy band, which in our case is $100-300 \mathrm{keV}$, to compute a time-dependent ratio. This ratio is subsequently applied to the background value, estimated by averaging the flux recorded during the quiet period (01:18:45-01:20:25 UT) in all of the energy bands, enabling us to derive a timedependent background evolution that then serves as an input to further spectral fitting. The background-subtracted X-ray spectrum is evidently above the background level only up to the energy $<12 \mathrm{keV}$. We thus fitted the background-subtracted spectra for each time interval with an isothermal photon model available within OSPEX. The fitted spectra are shown in Figure 6 for a few selected time intervals (panels (a)-(c)), while the temperature and emission measures of the flare plasma (panels (d) and (e)) are found to be varying in the range 7.7-9.1 MK and $0.9-3 \times 10^{48} \mathrm{~cm}^{-3}$, respectively. From Figure 6, it is seen that the X-ray photon flux beyond $10 \mathrm{keV}$ approximates the background flux, and hence the nonthermal characteristics of the flare plasma could not be determined with certainty from the spectral analysis.

\subsection{Polarizations of GMRT $610 \mathrm{MHz}$ Sources}

Figure 7 shows a sequence of SDO/HMI images overlaid by GMRT $610 \mathrm{MHz}$ radio source contours in pink. The top row shows the images in RCP, while the bottom row shows the images in LCP. The images from left to right show radio sources at the onset of the $610 \mathrm{MHz}$ flare at 04:10:25 UT, at the $610 \mathrm{MHz}$ flare peak at 04:11:05 UT, and after the $610 \mathrm{MHz}$ flare peak at 04:11:45 UT. Source LS has been found in both LCP and RCP images at all times having relatively strong emission in the LCP images. Thus, source LS is weakly left circular polarized throughout, with a degree of circular polarization $\mathrm{DCP}=\left(I_{R}-I_{L}\right) /\left(I_{R}+I_{L}\right)$ of $\sim 10 \%$, where $I_{R}$ and $I_{L}$ are the intensities of RCP and LCP, respectively. Source S1 

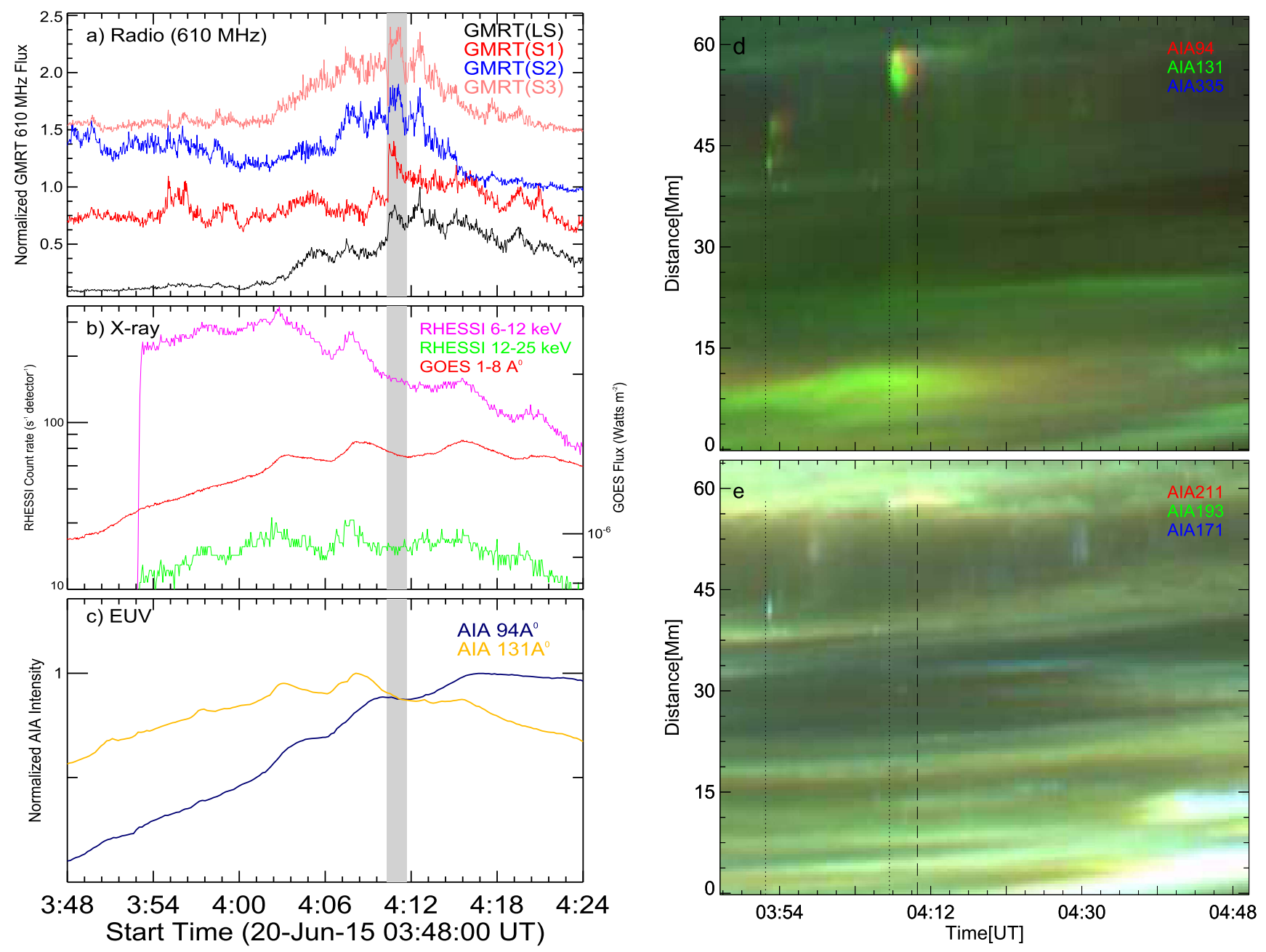

Figure 5. (a) Normalized radio fluxes of GMRT $610 \mathrm{MHz}$ sources LS, S1, S2, and S3. (b) GOES flux in the 1-8 A channel and RHESSI count rates in energy bands 6-12 and 12-25 keV. (c) Normalized EUV intensities in AIA hot passbands of 94 and $131 \AA$. The radio flare maximum, corresponding to our time of interest, is indicated by a vertical gray bar in panels (a)-(c). (d) and (e) Distance-time maps of AIA hot and cold channel maps showing flare brightenings marked by vertical dotted lines at 03:52:20 UT and between 04:07:25 and 04:10:25 UT.

is seen in both LCP and RCP pre- and post-burst images; it is, however, found only in RCP images during the burst, located above the region of negative magnetic polarity. Thus, source $\mathrm{S} 1$ is strongly right circular polarized, having a DCP of $\sim 50 \%$.

Sources S2 and S3, on the other hand, have been observed only in the burst images. Source S3 is seen only in LCP burst images with a DCP of $\sim 60 \%$, located above the regions of positive magnetic polarity. Source S2 is found in both RCP and LCP burst images with a DCP of $\sim 10 \%$ and having relatively strong emission in LCP images. Hence, it is weakly left circularly polarized. The strong polarization of sources S1 and S3 indicates that they are likely associated with fundamental radiation, while the weak polarization of sources LS and S2 indicates that they are possibly due to harmonic radiation.

\subsection{Magnetic Field Configuration of GMRT $610 \mathrm{MHz}$ Sources}

In order to understand the nature and origin of GMRT $610 \mathrm{MHz}$ radio sources, their spatial image locations are compared with the coronal magnetic fields. The coronal magnetic fields were computed using photospheric magnetic fields from an HMI magnetogram at 04:11:05 UT as input to a potential field source surface extrapolation (PFSS; Schrijver \&
De Rosa 2003). The computed extrapolated magnetic fields are overplotted on background HMI and AIA $94 \AA$ images, as shown in Figure 8. For clarity, only a few coronal magnetic field lines are plotted. The magnetic field lines fan out from the magnetic active region AR12371 into the corona, forming either the closed loops (indicated by white lines) or the open field lines (indicated by green lines). The smaller loops are mostly located near the active region, while the larger ones spread out in all directions with one of their ends rooted in the active region. The contours of GMRT radio sources (in blue) are overplotted on the background magnetogram and EUV images.

It is noticed that source $\mathrm{S} 2$ is located near the loop-top region of a loop, labeled as L1 in Figure 8 and shown as a pink curve, one of whose ends is at the positive polarity region P1 of active region AR12371, while the other end is at the negative polarity region $\mathrm{N} 2$ of active region AR12371. While source LS is located near the loop-top region of a very long loop, labeled as L2 in Figure 8 and shown as a pink curve, with one of its ends rooted in active region AR12371, near the positive polarity region $\mathrm{P} 1$, and the other end located far away somewhere near the southeast limb. Although there could be other loops located along the LOS of GMRT radio sources S2 and LS, we conclude 

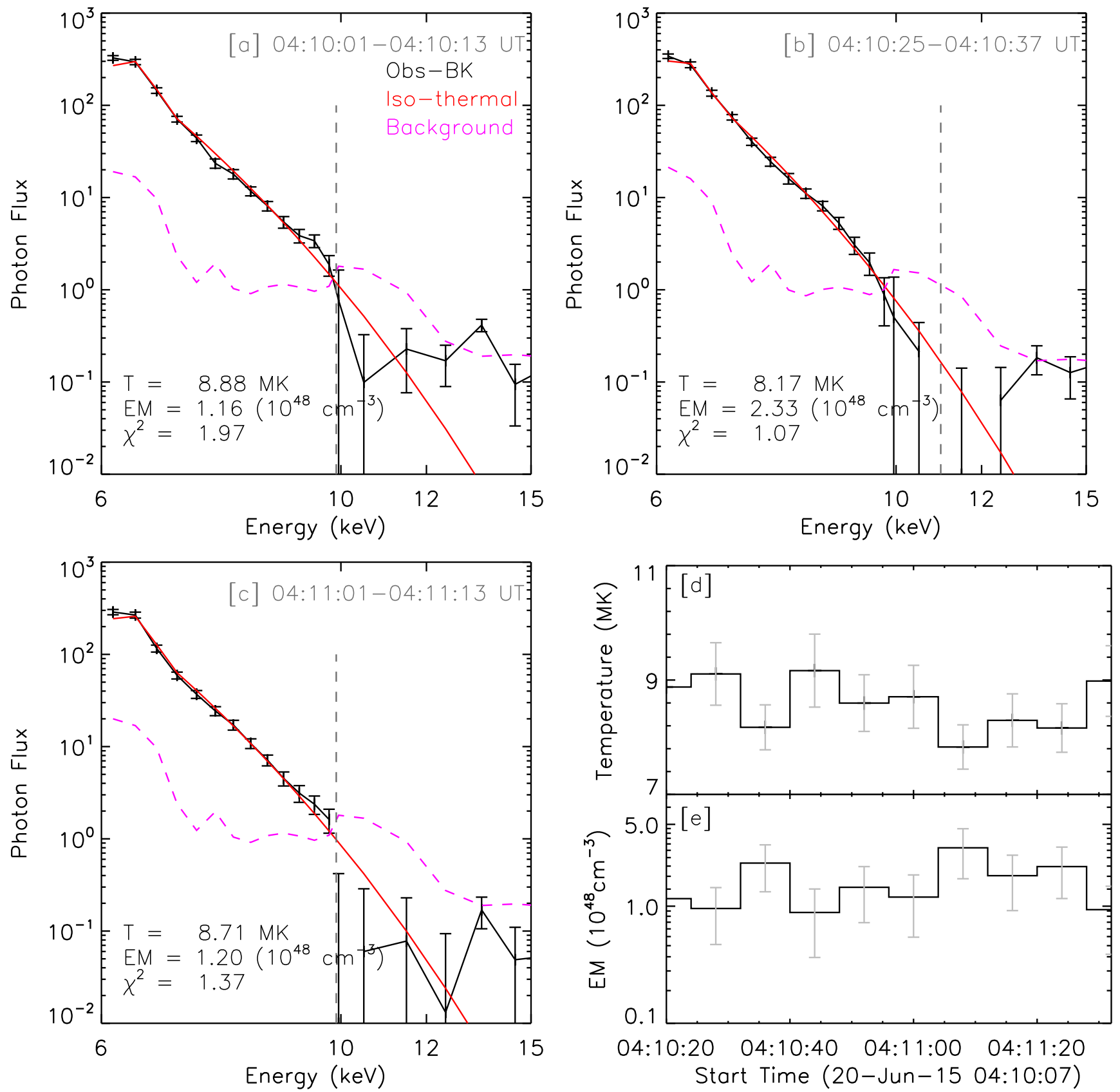

Figure 6. (a)-(c) RHESSI background-subtracted X-ray spectra (black) above $6 \mathrm{keV}$ for a few selected time intervals during 04:10-04:12 UT, corresponding to the radio flare maximum. In each panel, the dotted pink curve is the background of spectrum, while the forward fit to the background-subtracted spectrum, using an isothermal model, is shown by a solid red curve. In all panels, the vertical line indicates the high-energy end up to which the spectra are deemed to be fitted. Also indicated are the values of temperature, emission measure, and goodness of fit (measured by $\chi^{2}$ ). (d) and (e) Temperature and emission measure of the flare emission determined from the fitted X-ray spectra during the time interval 04:10-04:12 UT.

that the two sources are possibly from the loop-top region. By comparing the extrapolation results, we conclude that sources $\mathrm{S} 1$ and S3 are possibly from the legs of the flaring loop. This is implied from their positions near the ends of the extrapolated loop, shown by a pink curve, that lies on the hot flare loops as seen in the AIA $94 \AA$ image of Figure 8 (right panel). The extrapolated loop, intersecting sources $\mathrm{S} 1$ and $\mathrm{S} 3$, is also originated in the positive polarity region $\mathrm{P} 1$. Overall, the magnetic field configurations associated with all four GMRT sources possibly have a common origin. It is further noted that the open field lines anchored near the positive polarity region P1 appear to be strongly divergent at larger coronal heights.
Using the PFSS extrapolations and a coronal density model (see Appendix A), we estimate the heights of the sources S2 and LS, which are, respectively, $\sim 58 \mathrm{Mm}$ or $1.08 R_{s}$ and $\sim 127 \mathrm{Mm}$ or $1.18 R_{s}$. The sources S1 and S3 are found at the height of $\sim 23 \mathrm{Mm}$ or $1.02 R_{s}$ (see Appendix A). From the PFSS extrapolation, we estimated the coronal magnetic field strengths at the locations of GMRT sources S1, S2, S3, and LS, which are found to be about $72,40,127$, and $1.3 \mathrm{G}$, respectively. Since we obtained the heights and magnetic field strengths at the location of GMRT sources using the density values from the coronal density model and the PFSS extrapolations, we computed the ratio of fundamental plasma 

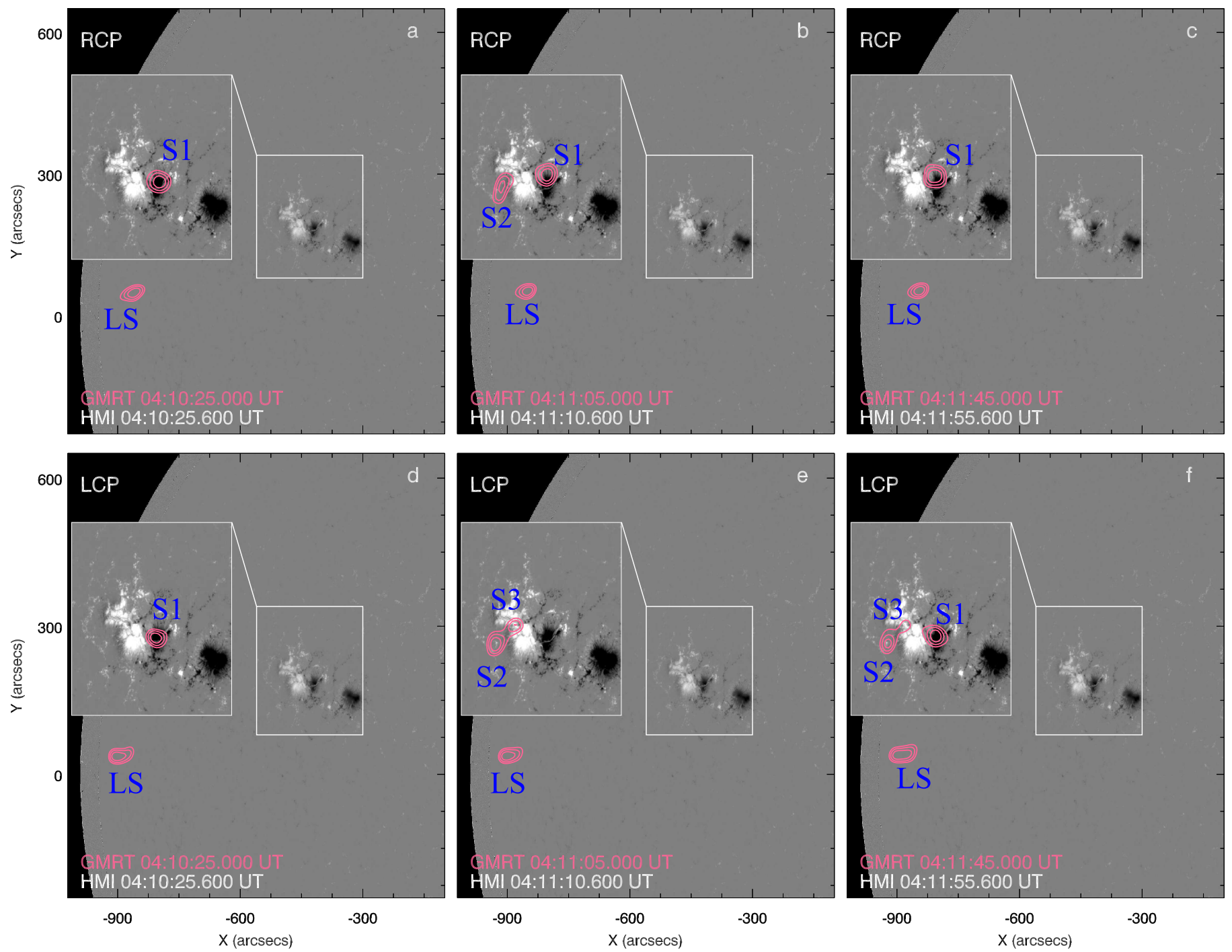

Figure 7. Sequence of $S D O / \mathrm{HMI}$ images overlaid by GMRT $610 \mathrm{MHz}$ source contours (pink) at RCP (top row) and LCP (bottom row). The columns, from left to right, show GMRT contour images at the onset of the $610 \mathrm{MHz}$ flare at 04:10:25 UT, at the 610 MHz flare peak at 04:11:05 UT, and after the 610 MHz flare peak at 04:11:45 UT. Source LS is seen throughout in both RCP and LCP images, while source S1 is seen in both RCP and LCP images during pre- and post-burst periods and only in LCP images during the burst. Sources S2 and S3 first appeared during the burst, with S2 seen in both RCP and LCP images, while S3 is seen only in RCP images.

frequency $\left(f_{p}=8980 \sqrt{n_{e}}\right)$ to gyrofrequency $\left(f_{b}=\frac{e B}{2 \pi m_{e}}\right)$ and found a value $>1$ for all of the GMRT sources.

\subsection{Metric Dynamic Spectra}

The decimetric dynamic spectra (figure not shown here) from the SBRS/YNAO in the frequency range $625-1500 \mathrm{MHz}$ and the Miangtu Spectra Radio Heliograph (MUSER; Yan et al. 2012) in the frequency range $400-2000 \mathrm{MHz}$ were examined. We also checked the radio dynamic spectra from the publicly available e-callisto network of solar radio spectrometers database. There appeared to be no evidence of Type III bursts in the decimetric frequency range covering the frequency of $610 \mathrm{MHz}$ during the radio flare maximum. However, metric Type III bursts were identified in the dynamic spectra of SBRS/YNAO during this period. Figure 9(a) shows YNAO metric spectra from 04:09:54 to 04:11:42 UT depicting the occurrence of Type III bursts. The upper and lower panels of Figure 9(a), respectively, show the spectra of LCP and RCP. Many Type III bursts with different start frequencies and bandwidths are observable from Figure 9(a). One such Type III burst occurring at 04:10:23 UT is indicated by white arrows in Figure 9(a). It started at a high frequency compared to the other bursts, having a duration of $0.5 \mathrm{~s}$, bandwidth of $\sim 130 \mathrm{MHz}$, and negative frequency drift rate of $65 \mathrm{MHz} \mathrm{s}^{-1}$. Note that the GMRT decimetric sources S1, S2, S3, and LS showed a quasiperiodic burst peak that started just at this time.

For a clear view of the Type III bursts, their light curves at a few selected frequencies are depicted in Figure 9(b). A few Type III bursts with high start frequencies are indicated by numbers in Figure 9(b). Also, bright narrowband metric burst emission has been noticed in the frequency range 200-220 MHz between 04:10:48 and 04:11:24 UT, corresponding to the $610 \mathrm{MHz}$ radio flare peak from both LCP and RCP spectra shown in Figure 9(a), and is indicated by red arrows. The spectra and light curves of the Type III burst of interest occurring at 04:10:23 UT, indicated by the white arrows in Figure 9(a) and demarcated between the vertical dotted lines in Figure 9(b), have been shown at the few selected 

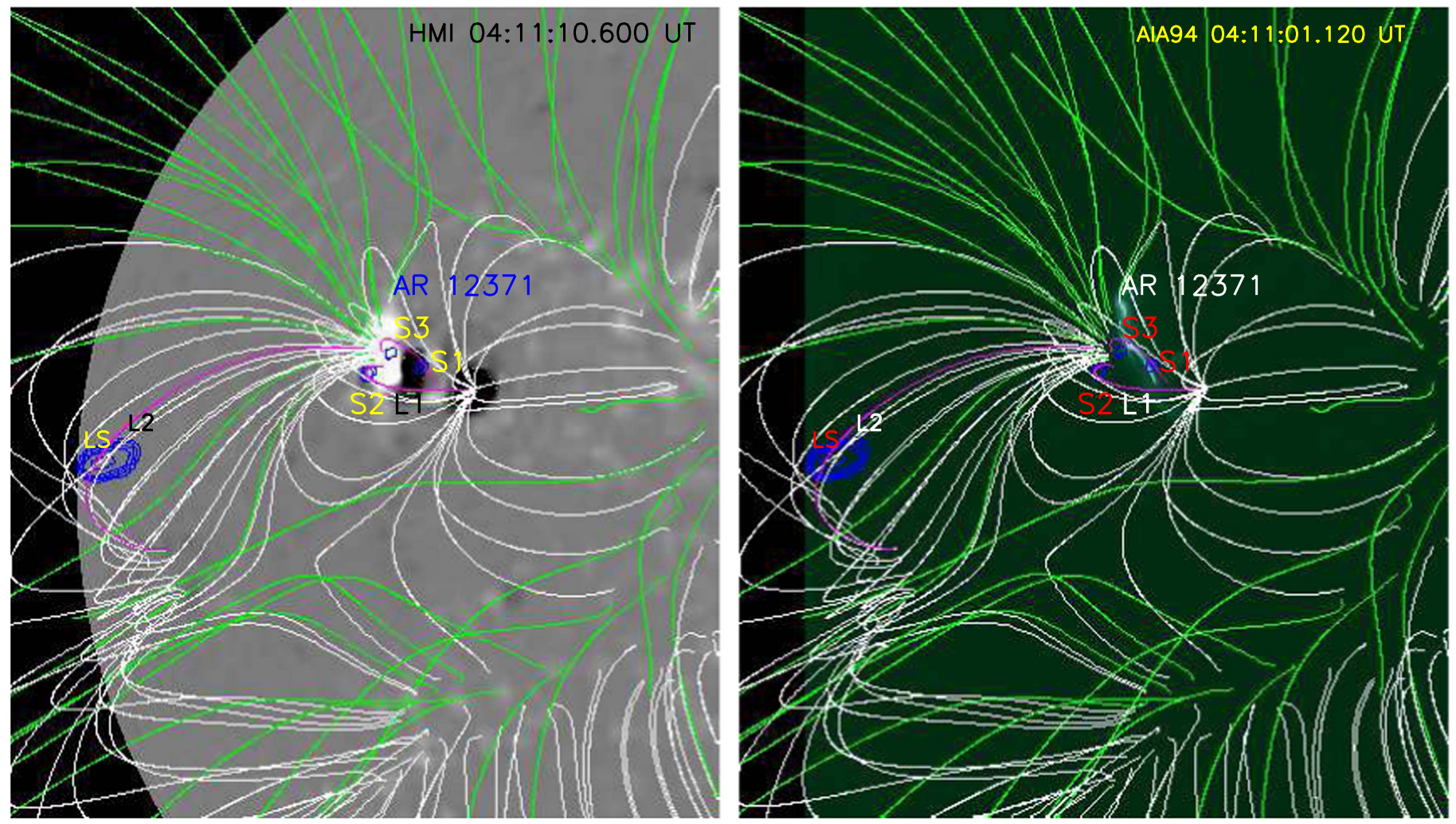

Figure 8. The PFSS computed coronal magnetic field lines of closed loops (white) and open fields (green) overlaid on background images of SDO/HMI (left panel) and SDO/AIA $94 \AA$ A (right panel). Also, overplotted in blue are the contours of sources LS, S1, S2, and S3 imaged at GMRT 610 MHz. The magnetic loops associated with GMRT sources S2 and LS are shown by the pink curves labeled as L1 and L2, respectively.

clean frequency channels in Figures 9(c) and (d), depicting the negative frequency drift of the burst.

The Type III bursts are noticed in both LCP and RCP metric spectra (Figure 9(a)); however, they show strong emission in the LCP spectra. This is also the case for our Type III burst of interest, which shows strong emission in LCP (Figure 9(d)). Thus, the metric bursts are weakly left circularly polarized. The frequency drift rate of the Type III burst noticed at 04:10:23 UT was used (see Appendix B) to estimate the electron beam velocity, which we found to be $\sim 0.13 c$. The beam velocity of $\sim 0.13 c$ corresponds to a kinetic energy of $\sim 29 \mathrm{keV}$.

The temporal association of the SBRS/YNAO metric Type III bursts and the GMRT $610 \mathrm{MHz}$ burst activity is shown in Figure 10, which shows, in the top panel, the temporal profiles of GMRT sources LS and S1 from 04:10:12 to 04:11:42 UT during the $610 \mathrm{MHz}$ flare maximum. The middle panel shows the temporal profiles of GMRT sources S2 and S3, and the bottom panel shows the temporal profiles of the Type III bursts at the selected frequencies of the SBRS/YNAO metric spectra. Several Type III bursts are clearly observable in association with the rise of the GMRT burst activity. In fact, a strong Type III burst is noticed just at the start of the GMRT burst activity at 04:10:23 UT, indicated by the vertical dotted lines.

\section{Discussion}

We have presented in this paper the first high time cadence $(0.5 \mathrm{~s})$ solar radio maps imaged by GMRT at the frequency of $610 \mathrm{MHz}$ during two (a C1.4 and an M1.0) flares and a CME. The flares and CME took place on 2015 June 20 in active region AR12371. It is seen from the high spatial resolution and dynamic range images of GMRT $610 \mathrm{MHz}$ that the radio sources during the M1.0 flare and the CME onset are located just above the flaring active region. In contrast, the radio source, namely LS, during the C1.4 flare is located about $500^{\prime \prime}$ from the flaring active region. This is unique, and, for the first time, to the best of our knowledge, a radio burst source during a flare emission is reported in the decimetric wavelength range that is located so far from the flaring site.

As mentioned earlier, the location of radio sources away from the flaring active region has been commonly observed in metric frequencies. This can be understood from the fact that the radio sources at low frequencies are usually located higher in the corona (Pick \& Vilmer 2008). However, in the present case, the frequency of observation is in the decimetric range, but still the radio source LS is located even farther than the reported cases in the metric frequency range. The question of interest, therefore, is how is that possible? Weak radio burst sources $\mathrm{S} 1, \mathrm{~S} 2$, and $\mathrm{S} 3$ are also identified during the radio flare maximum of the C1.4 flare and are, unlike the radio source LS, located near the flaring active region.

Based on our multiwavelength analysis and PFSS magnetic field extrapolation, in the following, we discuss the question of interest, the location of the electron acceleration region, and the nature of the GMRT radio sources.

\subsection{The Electron Acceleration Region}

From our study, we have shown that all of the GMRT radio sources, widely separated in space, exhibit a close temporal correlation. Further, from our PFSS extrapolation, a common origin of the magnetic field lines intersecting the GMRT radio sources has been observed. All of the field lines originated in the positive polarity region $\mathrm{P} 1$ of the flaring active region. It led 

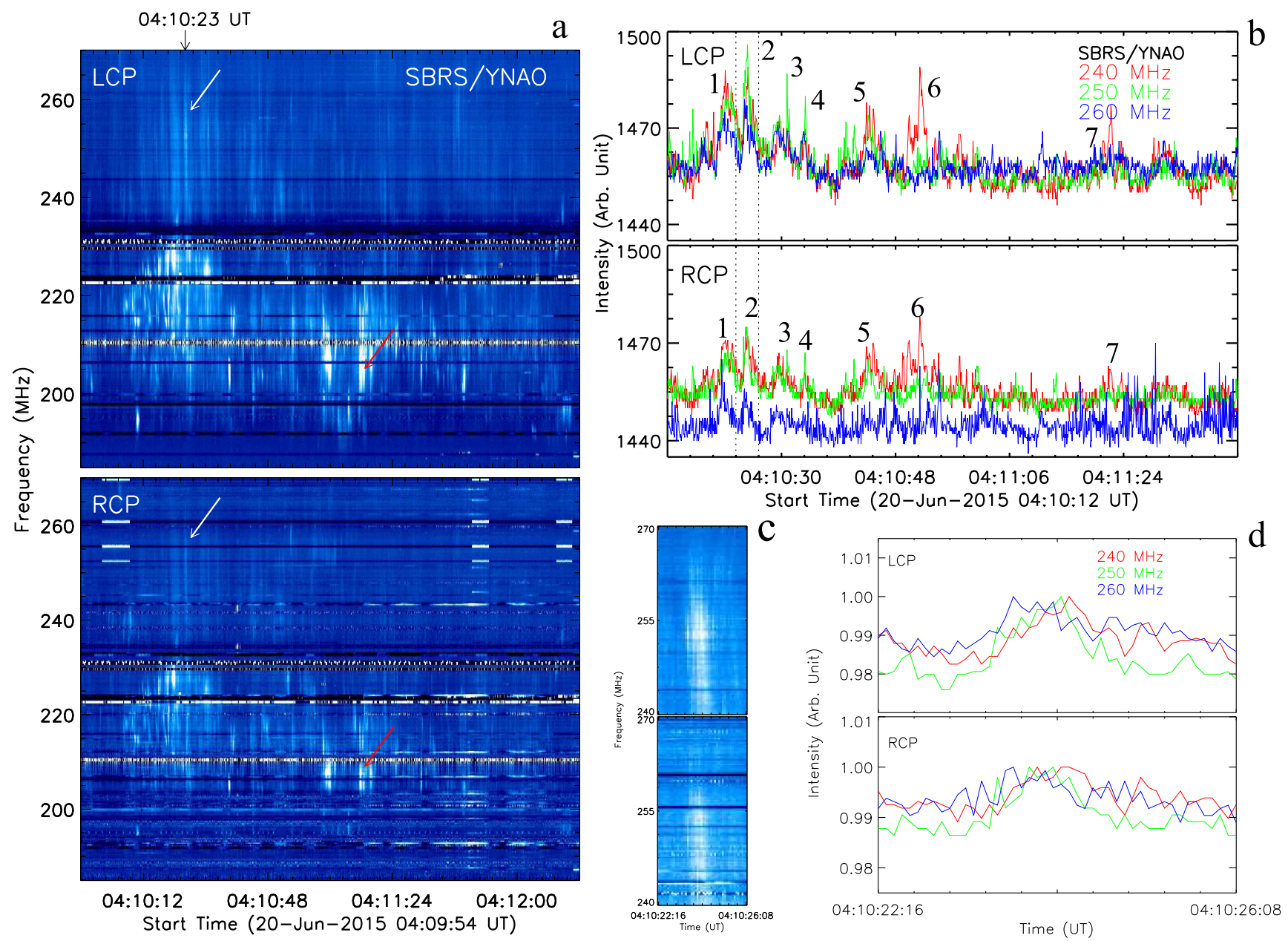

Figure 9. (a) Enhanced view of metric radio dynamic spectra from SBRS/YNAO between 04:09:54 and 04:11:42 UT in LCP (top) and RCP (bottom), depicting the Type III burst of interest and other narrowband Type III bursts. The Type III burst of interest at 04:10:23 UT is marked by arrows. (b) Light curves at selected frequencies depict the drift of bursts from higher to lower frequencies. (c) and (d) Enhanced view of the spectra and light curve, respectively, of the Type III burst of interest.

us to suggest that the source electrons or the exciters of the GMRT radio sources originated from a common electron acceleration site, which is likely located near the flaring active region. It is known that the interaction sites of the small flaring loops and larger loops could serve as the preferred sites for electron acceleration and energy release (Pick \& Vilmer 2008). So, the energetic electrons could originate from a common interaction site of the low-lying flare loops and high-arching loops. The accelerated electrons from this site were injected simultaneously into different magnetic field lines, which subsequently propagated along the field lines and emitted at the respective locations of the GMRT radio sources.

Also, the observed temporal association of the metric Type III bursts and the GMRT radio burst activity apparently indicates that they can be causally related. This suggests that the exciters of the radiating electrons, resulting in the metric Type III bursts and GMRT burst activity, originated from the same electron acceleration site. In other words, the open field lines in which the Type III excited electrons propagate and the closed loops in which the exciting agent of GMRT radiating electrons travels could be interacting near a common interaction site. From our PFSS extrapolations, we have seen the divergent open field lines located above the positive polarity region $\mathrm{P} 1$ of the flaring active region. It is known that the open field lines usually appear divergent (Aschwanden et al. 1992) due to the propagation of accelerated electrons along them. This suggests that the electrons may originate from an acceleration site located in the lower corona. It further supports that the open field lines and closed loops could be interacting at a common site located near the flaring active region where the radiating electrons responsible for the bursts originated.

\subsection{The Emission Mechanism}

All of the GMRT radio sources show high brightness temperatures, $>10^{11} \mathrm{~K}$, suggesting that they are generated by a coherent emission mechanism, that is, a plasma or an ECM radiation process. For ECM emission to be possible, $f_{p}$ must be less than $f_{b}$ at the emission site. Instead, based on a coronal density model and PFSS magnetic field extrapolations, we have found a ratio $f_{p}$ to $f_{b}>1$ for all of the GMRT sources at their respective observed locations. This suggests that the GMRT sources could be due to a plasma emission process.

Also, from GMRT polarization maps, we have shown that sources S1 and S3 are probably due to fundamental radiation, while sources S2 and LS are likely to be harmonic radiation. The estimated density scale heights for a frequency of $610 \mathrm{MHz}$ for fundamental and harmonic radiation, as obtained using a coronal electron density model by Aschwanden \& Benz (1995), would be 23 and $41 \mathrm{Mm}$, respectively. Thus, the 


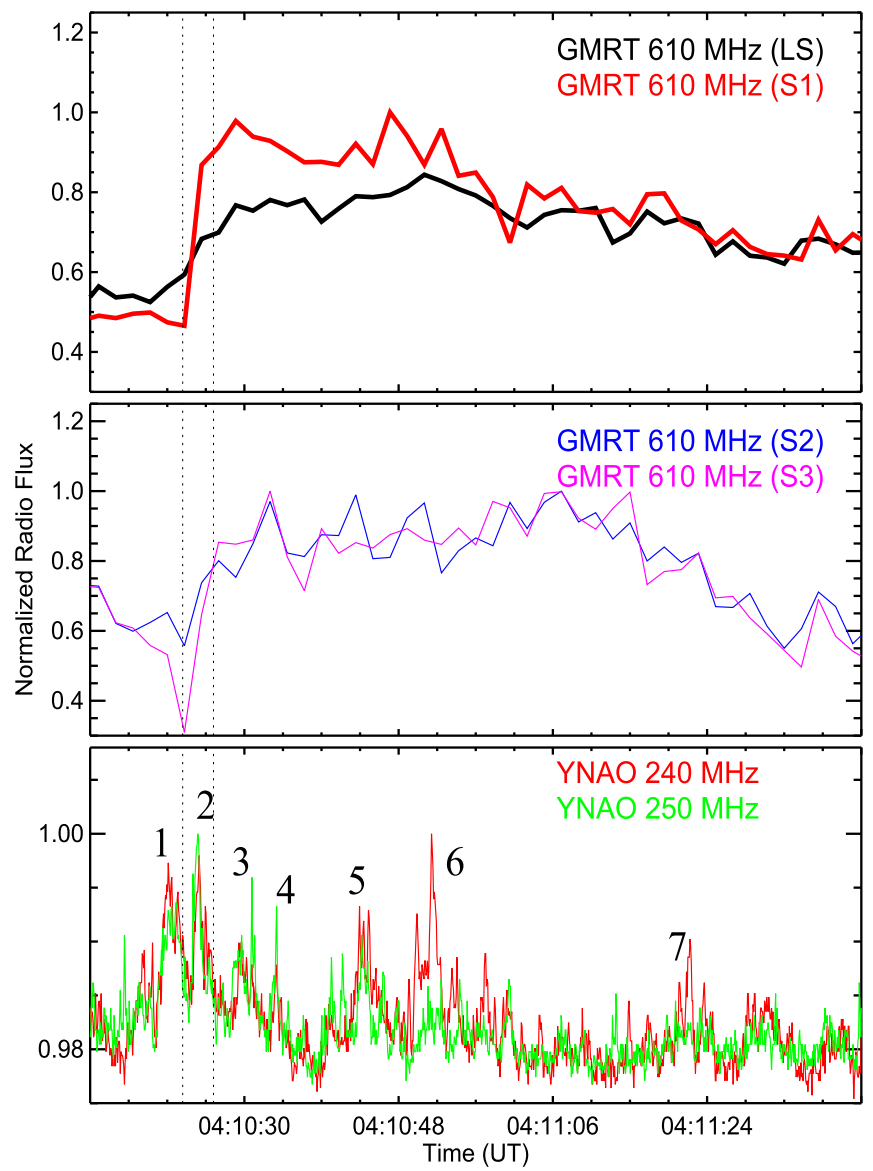

Figure 10. Shows the normalized radio fluxes of GMRT sources LS and S1 (top panel), GMRT sources S2 and S3 (middle panel), and Type III bursts at the selected frequencies of SBRS/YNAO (bottom). Several Type III bursts, numbered from 1 to 7 , are noticed in the metric frequencies of SBRS/YNAO associated with the rise of the GMRT $610 \mathrm{MHz}$ burst activity. Also, indicated between the vertical dotted lines is a strong Type III burst, which is noticed around 04:10:23 UT, just at the start of the rising activity of the GMRT sources.

density scale height for sources S1 and S3 would be $23 \mathrm{Mm}$, while that for sources S2 and LS would be $41 \mathrm{Mm}$. However, the actual height estimates for sources S2 ( 58 Mm) and LS ( $\sim 127 \mathrm{Mm})$, obtained from comparing our images and PFSS extrapolations (see Appendix A), are different from their density scale height of $41 \mathrm{Mm}$. Hence, one needs to normalize the Aschwanden density model by a suitable multiplication factor so as to reconcile the density scale height with the height obtained from the PFSS extrapolation. It is found that a twofold Aschwanden electron density model could explain the estimated height for the harmonic source S2. Thus, the normalized estimated heights using the twofold Aschwanden electron density model for the fundamental sources S1 and S3 would be $\sim 28 \mathrm{Mm}$.

Even if we assume that the source LS is a second harmonic emission source, the twofold Aschwanden density model cannot explain its estimated height from the PFSS extrapolation. This suggests that if the source LS is due to plasma radiation, it should be generated lower in the corona. The plasma radiation, however, could have escaped into the higher corona by a wave-ducting mechanism along a density-depleted tube. From our study, we have shown that the source LS is located on top of a high-arching loop with one of its ends anchored near the flaring active region. The high-arching loop could have acted as a density-depleted duct. Also, as mentioned earlier, a common electron acceleration site is located near the flaring active region. This suggests that the flare-associated upward-streaming electrons inside the tube could have produced the source LS by a plasma emission process somewhere near the flaring active region. If this is the case, then the requirement of ECM condition $f_{p}<f_{b}$ (Morosan et al. 2016) may also be satisfied, considering the much stronger magnetic field in the core region of the AR. Thus, ECM may also be a viable mechanism for the source LS.

A possible ECM process was proposed by Wang (2015), who suggested that flare-associated streaming electrons, while propagating in a density-depleted tube, can be pitch-anglescattered by enhanced turbulent Alfvén waves. As a result, the electrons exhibit a velocity distribution that is unstable to ECM instability. It is likely that the flare-associated upwardstreaming electrons in this case, after being pitch-anglescattered by the turbulent Alfvén waves, could have resulted in an ECM instability. The instability, in turn, would have generated $\mathrm{o}$ - or $\mathrm{x}$-mode electromagnetic waves near the second harmonic of the gyrofrequency, that is, at $\sim 610 \mathrm{MHz}$. Hence, the source LS could be generated near the flaring site either by a plasma or by an ECM radiation process. However, it could have escaped into the higher corona due to the wave-ducting effect.

On the other hand, unlike the source LS, which has been identified throughout the radio flare, the sources S1, S2, and S3 have been identified at the radio flare maximum. As estimated in our study from a RHESSI spectral analysis, we have found that the temperature of the ambient plasma during that period has been high, that is, $8-9 \mathrm{MK}$. Hence, the free-free absorption would be low, allowing the detection of radio sources S1, S2, and S3. One can argue the possibility of ECM emission for the sources $\mathrm{S} 1, \mathrm{~S} 2$, and $\mathrm{S} 3$, so we recomputed the ratio $f_{p}$ to $f_{b}$ based on their normalized heights using the twofold Aschwanden electron density model at their respective observed locations. But, we found a ratio $>1$. This suggests that the GMRT sources $\mathrm{S} 1, \mathrm{~S} 2$, and S3 were likely due to a plasma emission process.

\subsection{The Wave Mode}

It is known that fundamental plasma emission is highly circularly polarized in the sense of the o-mode, as the X-mode cannot escape above a cutoff frequency of $f_{p}+f_{b} / 2$ (Kundu et al. 2006; Nindos et al. 2008). Harmonic plasma emission is weakly polarized; however, it is often polarized in the sense of o-mode (Nindos et al. 2008). It is found that, except for the source LS, the GMRT sources $\mathrm{S} 1, \mathrm{~S} 2$ and $\mathrm{S} 3$ are by a plasma emission process, hence, they could be polarized in the sense of o-mode. From our study, we have shown that sources S1 and S3 are, respectively, strongly RCP and LCP, and the directions of the magnetic fields from the PFSS extrapolation in sources $\mathrm{S} 1$ and S3 are, respectively, downward- and upward-directed. Thus, the dominant wave mode of sources S1 and S3 would be o-mode. Source S2 is, on the other hand, weakly LCP and located on the loop with one of its ends anchored in regions of upward-directed magnetic field lines. Thus, the dominant wave mode of source S2 would be o-mode. The metric Type III bursts reported in the paper are also weakly LCP bursts. Thus, the dominant wave mode of the Type III bursts will be o-mode if their burst sources are also located in the regions of the upward-directed field lines. 
The source LS is weakly LCP. If we consider that it is due to plasma radiation, then the dominant wave mode of source LS would be o-mode, provided it is located in the regions of the upward-directed field lines. However, if we consider that the source LS is due to ECM radiation, then the value of $f_{b}$ for the source LS at its generation site would be $\sim 305 \mathrm{MHz}$. It is known that the wave modes of ECM emission depend on the ratio of $f_{p}$ to $f_{b}$ at the true source location or the generation site (Melrose \& Dulk 1982; Sharma \& Vlahos 1984; Régnier 2015; Wang 2015). Wang (2015) also suggested that if the ratio $f_{p}$ to $f_{b}$ is in the range $0.4-1.4$, then only a harmonic o- or $\mathrm{x}$-mode structureless burst would be observed, instead of a fundamental and harmonic pair burst. However, for the present case, the value of $f_{p}$ at the generation site could not be determined. Therefore, the wave mode associated with the source LS is unclear if it is due to an ECM process.

\section{Summary and Conclusion}

In the present work, we have presented high temporal and spatial radio images produced using GMRT $610 \mathrm{MHz}$ observations obtained during a C1.4-class solar flare on 2015 June 20. We have reported a strong decimetric radio source located far from the flaring active region. Also, we have reported weak decimetric radio sources identified during the $610 \mathrm{MHz}$ flare maximum. The weak radio sources are, however, located near the flaring site. Further, they show a close temporal correlation with the strong radio source and the metric Type III radio bursts identified in the SBRS/YNAO metric dynamic spectra.

Based on our investigation of a multiwavelength analysis and PFSS extrapolations, we have suggested that the source electrons of decimetric radio sources and metric Type III bursts were originated from a common electron acceleration site. The acceleration site is likely located near the flaring active region. In addition, we have argued that the observed weak radio sources are o-mode waves produced by a plasma emission process. The strong decimetric source, on the other hand, can be generated either by a plasma or by an ECM emission process. Its location far from the flaring site is presumably caused by the wave ducting of the emitted coherent radio waves that escaped along a density-depleted high-arching loop.

The single-frequency GMRT $610 \mathrm{MHz}$ observations provide important insights on the flare-accelerated electrons. However, they are unable to track the streaming electrons associated with the electron acceleration process, because they traverse a wide range of coronal heights as they propagate out from the electron acceleration site. We expect that future high-cadence (25-200 ms), high spatial resolution $(1 "$ '3-50"), and multifrequency $(0.4-2 \mathrm{GHz})$ imaging observations from MUSER can help in studying the details of electron beam trajectories and provide useful insights into the source regions of electron acceleration at decimetric frequencies.

The authors thank the staff of the GMRT for help during the observations. The authors also acknowledge the SDO/AIA, SDO/HMI, NASA/RHESSI, and GOES teams for making the data publicly available. SKB is supported by the PIFI (Project No. 2015PM066) program of the Chinese Academy of Sciences and the NSFC (Grant No. 117550110422). L.J.C. and G.G. acknowledge support by the NSFC (Grant Nos. 11573043, 1143306 and 11403099, respectively). S.K.B. acknowledges scientific input from P. Subramanian, Baolin Tan, and Jie Huang.

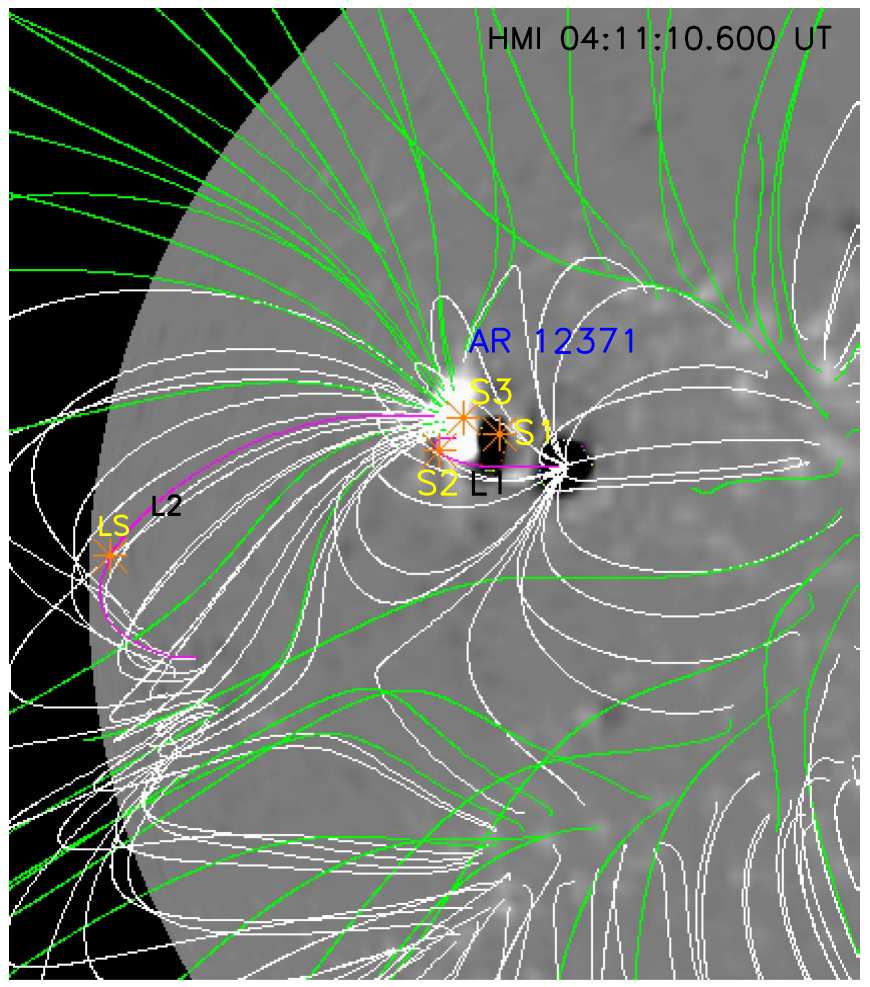

Figure 11. The PFSS computed coronal magnetic field lines are shown in white (closed field lines) and green (open field lines) overlaid on a background HMI image at the radio flare maximum. Overplotted as star symbols in orange are the centroids of the GMRT $610 \mathrm{MHz}$ sources LS, S1, S2, and S3. The magnetic field loops associated with sources S2 and LS are shown by the pink curves, labeled as L1 and L2

In addition, the authors thank the reviewer for constructive comments and suggestions that significantly improved the paper.

\section{Appendix A \\ Estimation of Heights of GMRT Radio Sources}

The comparison of spatial locations of GMRT radio sources with PFSS computed coronal magnetic fields showed that they were loop-top radiation (see Figure 8). In order to estimate the radial heights of the GMRT radio sources, we identified the coronal loop, obtained from PFSS extrapolation, on which the centroids of the GMRT radio sources were actually located, and the apex of the loop was situated close to the GMRT radio source. Figure 11 shows the PFSS computed coronal magnetic field lines overlaid on the background HMI magnetogram at 04:11:05 UT. The centroids of all GMRT radio sources (S1, S2, S3, and LS) are overplotted on the magnetogram as star symbols. It is seen from Figure 11 that the centroids of sources S2 and LS are located on the coronal loops, labeled as L1 and L2 and shown by the pink curves, whose loop apexes were close to the source centroids of the former. The apexes of loops L1 and L2, as estimated from PFSS extrapolation, were located at heights of $\sim 58$ and $\sim 140 \mathrm{Mm}$, respectively. The estimated heights are radial heights from the solar surface. Thus, the radial heights of GMRT sources S2 and LS could be $\sim 58 \mathrm{Mm}$ or $1.08 R_{s}$ and $\sim 140 \mathrm{Mm}$ or $1.2 R_{s}$, respectively.

It may, however, happen that the apex of the loop can be located at a nonradial position. The actual height of the radio sources then could be different, which could only be estimated by knowing the inclination of the loop apex to the LOS direction from Earth. To find the inclination of the loop apex, 
the views of the loop from two different angles, such as $S D O$ (Earth's view) and the Solar Terrestrial Relation Observatory (STEREO), should be available. During this event, however, the twin spacecraft STEREO A and B were separated from the Sun-Earth line by angles of $177^{\circ}$ and $172^{\circ}$, respectively, making it difficult to find the inclination. From the images, however, the apparent inclination could be estimated (Iwai et al. 2014) and was $\leqslant 2^{\circ}$ for source $S 2$ and about $25^{\circ}$ for source LS. Thus, the actual heights of GMRT source S2 would be the same as the radial heights of $\sim 58 \mathrm{Mm}$ or $1.08 R_{s}$, while the actual height of source LS would be $127 \mathrm{Mm}$ or $1.18 R_{s}$.

It is found that sources S1 and S3 are located in the loop legs; we thus estimated the density scale heights for sources S1 and S3 using a coronal electron density model by Aschwanden \& Benz (1995). In the Aschwanden \& Benz (1995) density model, the electron density in the lower corona (for $h<160 \mathrm{Mm}$ ) can be obtained using the equation

$$
n_{e}=n 1 \cdot\left(\frac{h}{h 1}\right)^{(-p)} \text {, }
$$

where $n 1=n Q . \exp (-p), p=2.38, \lambda=6.9 \times 10^{10} \mathrm{~cm}, h 1=$ $1.6 \times 10^{10} \mathrm{~cm}, n_{Q}=4.6 \times 10^{8} \mathrm{~cm}^{-3}$, and $n_{e}$ is known from the frequency of observation by the relation $f=\mathrm{s} .8980 \sqrt{n_{e}}$ ( $\mathrm{s}=1$ for sources $\mathrm{S} 1$ and $\mathrm{S} 3$ ). Thus, the estimated density scale heights of sources $\mathrm{S} 1$ and $\mathrm{S} 3$ would be $\sim 23 \mathrm{Mm}$ or $1.02 R_{s}$.

\section{Appendix B \\ Estimation of Velocity of Type III Electron Beam}

The velocity of the electron beam can be estimated from the frequency drift rate, $\frac{d f}{d t}$, of the Type III burst and knowing the scale height, $H_{n}$, at the drift rate measured frequency $f$ :

$$
v \equiv 2 H_{n} \cdot \frac{1}{f} \cdot \frac{d f}{d t} .
$$

The $H_{n}$ is estimated using the St. Hilarie density model (Saint-Hilaire et al. 2013) knowing $n_{e}$ at the measured frequency, $f$, which is $\sim 2.2 \times 10^{8} \mathrm{~cm}^{-3}$. The St. Hilarie model is chosen because it is a solar wind-like density model of the form $n_{e}(r)=C\left(h / R_{s}\right)^{-2}$, where $h$ is heliocentric distance and $C$ is a normalization constant with a value of $1.2 \times 10^{6} \mathrm{~cm}^{-3}$. Thus, the estimated apparent velocity of the electrons was found to be $\sim 0.16 c$. To estimate the real velocity of the electrons, we need the angle of propagation between the beam direction and the LOS. However, we did not know the angle, and, without knowing the angle, the minimum possible real electron velocity, $v_{\min }$, could be estimated following the method described in Carley et al. (2016):

$$
v_{\min }=\frac{c v}{c+v} \text {. }
$$

The real velocity of the electron beam was therefore $\sim 0.13 c$.

\section{ORCID iDs}

Susanta Kumar Bisoi 자 https://orcid.org/0000-0002-9448-1794 P. Janardhan (1) https://orcid.org/0000-0003-2504-2576 Arun Kumar Awasthi $\mathbb{*}$ () https://orcid.org/0000-0001-5313-1125

\section{References}

Aschwanden, M. J. 2002, SSRv, 101, 1

Aschwanden, M. J. 2005, Physics of the Solar Corona. An Introduction with Problems and Solutions (2nd ed.; Berlin: Springer)

Aschwanden, M. J., Bastian, T. S., Benz, A. O., \& Brosius, J. W. 1992, ApJ, 391,380

Aschwanden, M. J., \& Benz, A. O. 1995, ApJ, 438, 997

Bastian, T. S., Benz, A. O., \& Gary, D. E. 1998, ARA\&A, 36, 131

Benz, A. O. 1985, SoPh, 96, 357

Benz, A. O. 2004, in Solar and Space Weather Radiophysics, ed. D. E. Gary \& C. U. Keller (Berlin: Springer), 203

Benz, A. O. 2008, LRSP, 5, 1

Benz, A. O., Barrow, C. H., Dennis, B. R., et al. 1983, SoPh, 83, 267

Benz, A. O., Jaeggi, M., \& Zlobec, P. 1982, A\&A, 109, 305

Benz, A. O., Saint-Hilaire, P., \& Vilmer, N. 2002, A\&A, 383, 678

Carley, E. P., Vilmer, N., \& Gallagher, P. T. 2016, ApJ, 833, 87

Chen, B., Bastian, T. S., Shen, C., et al. 2015, Sci, 350, 1238

Chen, B., Bastian, T. S., White, S. M., et al. 2013, ApJL, 763, L21

Droege, F. 1977, A\&A, 57, 285

Dulk, G. A. 1985, ARA\&A, 23, 169

Duncan, R. A. 1979, SoPh, 63, 389

Fletcher, L., Dennis, B. R., Hudson, H. S., et al. 2011, SSRv, 159, 19

Gao, G., Wang, M., Dong, L., Wu, N., \& Lin, J. 2014, NewA, 30, 68

Hurford, G. J., Schmahl, E. J., Schwartz, R. A., et al. 2002, SoPh, 210, 61

Iwai, K., Shibasaki, K., Nozawa, S., et al. 2014, EP\&S, 66, 149

Karlický, M. 2004, A\&A, 417, 325

Karlický, M., \& Bárta, M. 2007, A\&A, 464, 735

Karlický, M., Fárník, F., \& Mészárosová, H. 2002, A\&A, 395, 677

Kliem, B., Karlický, M., \& Benz, A. O. 2000, A\&A, 360, 715

Kontar, E. P., Yu, S., Kuznetsov, A. A., et al. 2017, NatCo, 8, 1515

Kundu, M. R., White, S. M., Garaimov, V. I., et al. 2006, SoPh, 236, 369

Lemen, J. R., Title, A. M., Akin, D. J., et al. 2012, SoPh, 275, 17

Lin, R. P., Dennis, B. R., Hurford, G. J., et al. 2002, SoPh, 210, 3

Mann, G., Breitling, F., Vocks, C., et al. 2018, A\&A, 611, A57

Melrose, D. B. 1980, SSRv, 26, 3

Melrose, D. B. 1991, ARA\&A, 29, 31

Melrose, D. B., \& Dulk, G. A. 1982, ApJ, 259, 844

Mercier, C., Subramanian, P., Chambe, G., \& Janardhan, P. 2015, A\&A, 576, A136

Mercier, C., Subramanian, P., Kerdraon, A., et al. 2006, A\&A, 447, 1189

Mészárosová, H., Dudík, J., Karlický, M., Madsen, F. R. H., \& Sawant, H. S. 2013, SoPh, 283, 473

Morosan, D. E., Zucca, P., Bloomfield, D. S., \& Gallagher, P. T. 2016, A\&A, 589, L8

Nindos, A., Aurass, H., Klein, K.-L., \& Trottet, G. 2008, SoPh, 253, 3

Pesnell, W. D., Thompson, B. J., \& Chamberlin, P. C. 2012, SoPh, 275, 3

Pick, M., \& Vilmer, N. 2008, A\&ARv, 16, 1

Priest, E., \& Forbes, T. 2000, Magnetic Reconnection: MHD Theory and Applications (Cambridge: Cambridge Univ. Press)

Régnier, S. 2015, A\&A, 581, A9

Saint-Hilaire, P., Vilmer, N., \& Kerdraon, A. 2013, ApJ, 762, 60

Schou, J., Scherrer, P. H., Bush, R. I., et al. 2012, SoPh, 275, 229

Schrijver, C. J., \& De Rosa, M. L. 2003, SoPh, 212, 165

Sharma, R. R., \& Vlahos, L. 1984, ApJ, 280, 405

Sheridan, K. V., Labrum, N. R., \& Payten, W. J. 1972, NPhS, 238, 115

Slottje, C. 1980, in IAU Symp. 86, Radio Physics of the Sun, ed. M. R. Kundu \& T. E. Gergely (Dordrecht: Reidel), 195

Stewart, R. T. 1972, PASAu, 2, 100

Subramanian, P., Ananthakrishnan, S., Janardhan, P., et al. 2003, SoPh, 218,247

Subramanian, P., White, S. M., Karlický, M., et al. 2007, A\&A, 468, 1099

Swarup, G., Ananthakrishnan, S., Kapahi, V. K., et al. 1991, CSci, 60, 95

Tan, B. 2013, ApJ, 773, 165

Wang, C. B. 2015, ApJ, 806, 34

White, S. M., Benz, A. O., Christe, S., et al. 2011, SSRv, 159, 225

Wild, J. P., Murray, J. D., \& Rowe, W. C. 1953, Natur, 172, 533

Wild, J. P., \& Smerd, S. F. 1972, ARA\&A, 10, 159

Yan, Y., Zhang, J., Chen, Z., et al. 2012, IAUSS, 6, E2.16 